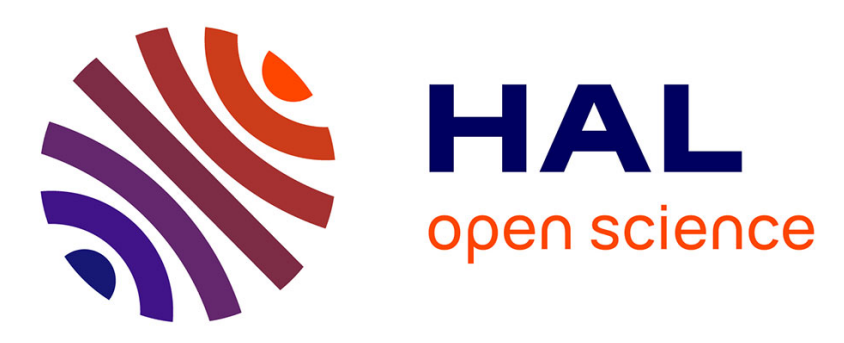

\title{
Estimation of the Degree of Polarization for Hybrid/Compact and Linear Dual-Pol SAR Intensity Images: Principles and Applications
}

Réza Shirvany, Marie Chabert, Jean-Yves Tourneret

\section{- To cite this version:}

Réza Shirvany, Marie Chabert, Jean-Yves Tourneret. Estimation of the Degree of Polarization for Hybrid/Compact and Linear Dual-Pol SAR Intensity Images: Principles and Applications. IEEE Transactions on Geoscience and Remote Sensing, 2013, vol. 51, pp.539-551. 10.1109/TGRS.2012.2202242 . hal-00778713

\section{HAL Id: hal-00778713 \\ https://hal.science/hal-00778713}

Submitted on 21 Jan 2013

HAL is a multi-disciplinary open access archive for the deposit and dissemination of scientific research documents, whether they are published or not. The documents may come from teaching and research institutions in France or abroad, or from public or private research centers.
L'archive ouverte pluridisciplinaire HAL, est destinée au dépôt et à la diffusion de documents scientifiques de niveau recherche, publiés ou non, émanant des établissements d'enseignement et de recherche français ou étrangers, des laboratoires publics ou privés. 


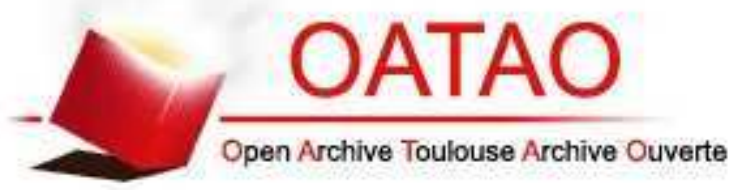

\section{Open Archive TOULOUSE Archive Ouverte (OATAO)}

OATAO is an open access repository that collects the work of Toulouse researchers and makes it freely available over the web where possible.

This is an author-deposited version published in : http://oatao.univ-toulouse.fr/ Eprints ID : 8086

To link to this article : DOI: 10.1109/TGRS.2012.2202242

URL : http://dx.doi.org/10.1109/TGRS.2012.2202242

To cite this version :

Shirvany, Reza and Chabert, Marie and Tourneret, Jean-Yves Estimation of the Degree of Polarization for Hybrid/Compact and Linear Dual-Pol SAR Intensity Images: Principles and Applications. (2013) IEEE Transactions on Geoscience and Remote Sensing, vol. 51 ( $\mathrm{n}^{\circ}$ 1). pp. 539-551. ISSN 0196-2892

Any correspondence concerning this service should be sent to the repository administrator: staff-oatao@ listes.diff.inp-toulouse.fr 


\title{
Estimation of the Degree of Polarization for Hybrid/Compact and Linear Dual-Pol SAR Intensity Images: Principles and Applications
}

\author{
Reza Shirvany, Student Member, IEEE, Marie Chabert, Member, IEEE, and \\ Jean-Yves Tourneret, Senior Member, IEEE
}

\begin{abstract}
Analysis and comparison of linear and hybrid/ compact dual-polarization (dual-pol) synthetic aperture radar (SAR) imagery have gained a wholly new importance in the last few years, in particular, with the advent of new spaceborne SARs such as the Japanese ALOS PALSAR, the Canadian RADARSAT-2, and the German TerraSAR-X. Compact polarimetry, hybrid dual-pol, and quad-pol modes are newly promoted in the literature for future SAR missions. In this paper, we investigate and compare different hybrid/compact and linear dual-pol modes in terms of the estimation of the degree of polarization (DoP). The DoP has long been recognized as one of the most important parameters characterizing a partially polarized electromagnetic wave. It can be effectively used to characterize the information content of SAR data. We study and compare the information content of the intensity data provided by different hybrid/compact and linear dual-pol SAR modes. For this purpose, we derive the joint distribution of multilook SAR intensity images. We use this distribution to derive the maximum likelihood and moment-based estimators of the DoP in hybrid/compact and linear dual-pol modes. We evaluate and compare the performance of these estimators for different modes on both synthetic and real data, which are acquired by RADARSAT-2 spaceborne and NASA/JPL airborne SAR systems, over various terrain types such as urban, vegetation, and ocean.
\end{abstract}

Index Terms-Compact polarimetry, degree of polarization (DoP), dual-pol, hybrid polarity, maximum likelihood (ML), multivariate gamma distribution (MGD), synthetic aperture radar (SAR).

\section{INTRODUCTION}

$\mathbf{P}$ OLARIMETRIC imagery is a well-established tool providing complementary information to traditional imagery in a variety of fields. Polarimetric imagery has been widely studied and employed in different applications such as astronomy [1], meteorological investigations [2], [3], wetland monitoring [4], estimation of forest parameters [5], oil spill detection [6], computer vision [7], and medicine [8].

Radar polarimetry has gained considerable importance in recent years. There exists a variety of spaceborne and airborne polarimetric synthetic aperture radar (SAR) systems;

The authors are with the University of Toulouse, IRIT/INP-ENSEEIHT/ TéSA, 31071 Toulouse, France (e-mail: reza.shirvany@enseeiht.fr; marie. chabert@enseeiht.fr; jean-yves.tourneret@enseeiht.fr). traditional ones are linear single-polarization (single-pol) and dual-polarization (dual-pol) systems. More sophisticated ones are full polarimetric systems. Other recently developed designs can be found in the literature, which are called hybrid/compact dual-pol and are proposed for future SAR missions. Singlepol SAR systems were designed using a single polarization; transmitting and receiving horizontally $(\mathrm{H})$ or vertically $(\mathrm{V})$ polarized radiation. In conventional dual-pol modes, two linear polarizations are considered, i.e., $(\mathrm{HH}, \mathrm{HV}),(\mathrm{VH}, \mathrm{VV})$, or $(\mathrm{HH}$, VV). Full polarimetric (full-pol) systems alternately transmit two orthogonal polarizations and record both received polarizations (HH, HV, VH, and VV). Full-pol SARs allow much more information to be extracted from a scene compared with single- and dual-pol data. However, they are disadvantaged by a lower radar swath coverage and higher antenna transmitter power requirements (see [9] and references therein for more details). As a result, hybrid/compact polarimetric systems have been widely investigated in recent years as a possible tradeoff in polarimetric SARs. hybrid/compact polarimetric imaging provides a wider swath width and, hence, greater area coverage, compared with a full-pol system. Souyris et al. [10] introduced the $\pi / 4$ mode, where the transmitted polarization is the superposition of linear horizontal and vertical polarizations $\left(L_{45^{\circ}}\right)$, and the received returns are recorded in both horizontal and vertical polarizations. In another study, Stacy and Preiss [11] proposed the dual circular polarimetric (DCP) mode based on a right (or left) circular polarization transmit, and right and left circular polarization receives (RR, RL). In a recent study, Raney [12] promoted a hybrid (circular linear) mode of operation (CL-pol), with a right (or left) circular polarization on transmission and two linear polarizations on reception (RH, RV).

It is important to understand the information content of different polarimetric images and to appreciate the suitable configuration for each particular application. Recent SAR systems can provide both complex and intensity data, whereas some other systems only provide amplitude data with no informative phase (e.g., airborne SAR (AirSAR) CYCLOPS and ENVISAT ASAR). The AirSAR CYCLOPS is an enhancement of the standard AirSAR quick-look processor, which is designed to provide amplitude imagery over a large range and azimuth swaths [13]. Such intensity data are of great interest for those investigators who require calibrated SAR data over large swaths, notably for monitoring large coastal and ocean areas under all-weather conditions. Analysis and comparison of 
different SAR imagery modes, particularly the comparison of linear and hybrid/compact modes, have gained new importance in the last few years [14], [15]. The aim of this paper is therefore to investigate in detail the information content of the intensity data in different hybrid/compact and linear dual-pol modes. The degree of polarization (DoP) can be effectively used to characterize the information content of SAR data. This scalar parameter is considered as the most important parameter characterizing a partially polarized electromagnetic wave [16], [17]. Knowledge of DoP can help to determine the nature of the objects that backscatter the wave. However, four intensity images are necessary for the classical estimation of the DoP; thus, estimation of the DoP based on only two (dual-pol) intensity images is a challenging task. This task is addressed in detail in this paper.

Images observed by SAR systems are degraded by speckle noise due to coherent interference of waves reflected from many elementary scatterers [9], [18]. This noise generates a grainy structure in the image and reduces the precision of the measurements. Speckle noise can be reduced by multilook processing where $q$-independent SAR images (of the same scene) are averaged to form a $q$-look image. The statistics of these multilook intensity images have been studied in particular cases (see [19] and [20] and references therein). In this paper, we derive the joint distribution of the multilook SAR intensity images in a more generalized case. Based on this distribution, we derive maximum likelihood (ML) and momentbased (MoM) estimators of the DoP in hybrid/compact and linear dual-pol modes. We evaluate and compare the performance of the proposed estimators for both synthetic and real data, which are acquired by RADARSAT- 2 spaceborne and NASA/JPL AirSAR systems. We compare the DoP estimators in different linear and hybrid/compact dual-pol SAR modes over various terrain types such as urban, vegetation, and ocean. Some potential applications of these DoP estimators are also presented.

This paper is organized as follows. In Section II, we first review some physical and mathematical definitions used throughout this paper and formulate the problem of interest. In Section III-A, the joint distribution of multilook SAR intensity images is derived. This distribution is used, in Sections III-C and III-D, to derive the ML and MoM estimators of the DoP. In Section IV, our synthetic and real data sets are described. Experimental results, discussions, and future work are presented in Section V, with concluding remarks given in Section VI.

\section{Polarization Characteristics}

Considering a right-handed system $\left(\hat{u}_{H}, \hat{u}_{V}, \hat{u}\right)$, an electromagnetic monochromatic plane wave propagating along $\hat{u}$ is expressed as

$$
\vec{E}(u, t)=\vec{E} e^{i(\omega t-\nu u)}
$$

where $\omega$ is the angular frequency, $\nu$ is the wavenumber, $\vec{E}=$ $E_{H} \hat{u}_{H}+E_{V} \hat{u}_{V}$ is a complex 2-D vector, and $\left(\hat{u}_{H}, \hat{u}_{V}\right)$ forms a basis with two orthogonal unit vectors. The polarization state of an electromagnetic wave is determined from the amplitudes of $E_{H}$ and $E_{V}$, and the relative phase between them. We note that $\vec{E}$, represented in vector form and denoted as $\boldsymbol{E}=$ $\left(E_{H}, E_{V}\right)^{T}$, is called the Jones vector [21]. In order to deal with a partially polarized wave, either the covariance matrix of $\boldsymbol{E}$ or the Stokes vector can be used. The covariance matrix of $\boldsymbol{E}$ is defined as [17], [22]

$$
\begin{aligned}
\Gamma=\mathrm{E}\left[\boldsymbol{E} \boldsymbol{E}^{\dagger}\right] & =\left(\begin{array}{cc}
\mathrm{E}\left[\left|E_{H}\right|^{2}\right] & \mathrm{E}\left[E_{H} E_{V}{ }^{*}\right] \\
\mathrm{E}\left[E_{V} E_{H}{ }^{*}\right] & \mathrm{E}\left[\left|E_{V}\right|^{2}\right]
\end{array}\right) \\
& \triangleq\left(\begin{array}{cc}
a_{1} & a_{3}+i a_{4} \\
a_{3}-i a_{4} & a_{2}
\end{array}\right)
\end{aligned}
$$

where E. is the expectation operator, ${ }^{\dagger}$ is the conjugate transpose, ${ }^{*}$ is the complex conjugate, and $|\cdot|$ denotes the magnitude of the complex field. In his remarkable paper in 1852, Stokes [23] introduced four measurable quantities, which are known as the Stokes parameters, for describing the properties of polarized light. Considering the Pauli group of matrices, i.e.,

$$
\begin{aligned}
& \boldsymbol{\sigma}_{0}=\left(\begin{array}{ll}
1 & 0 \\
0 & 1
\end{array}\right) \boldsymbol{\sigma}_{1}=\left(\begin{array}{cc}
1 & 0 \\
0 & -1
\end{array}\right) \\
& \boldsymbol{\sigma}_{2}=\left(\begin{array}{ll}
0 & 1 \\
1 & 0
\end{array}\right) \boldsymbol{\sigma}_{3}=\left(\begin{array}{cc}
0 & -i \\
i & 0
\end{array}\right)
\end{aligned}
$$

the multilook (empirical) covariance matrix $\bar{\Gamma}$ can be decomposed as [24], [25]

$$
\begin{aligned}
\overline{\boldsymbol{\Gamma}}=\left\langle\boldsymbol{E} \boldsymbol{E}^{\dagger}\right\rangle & =\left(\begin{array}{cc}
\left\langle\left|E_{H}\right|^{2}\right\rangle & \left\langle E_{H} E_{V}^{*}\right\rangle \\
\left\langle E_{V} E_{H}^{*}\right\rangle & \left\langle\left|E_{V}\right|^{2}\right\rangle
\end{array}\right) \\
& =\frac{1}{2}\left(\mathrm{~g}_{0} \boldsymbol{\sigma}_{0}+\mathrm{g}_{1} \boldsymbol{\sigma}_{1}+\mathrm{g}_{2} \boldsymbol{\sigma}_{2}+\mathrm{g}_{3} \boldsymbol{\sigma}_{3}\right) \\
& =\frac{1}{2}\left(\begin{array}{cc}
\mathrm{g}_{0}+\mathrm{g}_{1} & \mathrm{~g}_{2}-i \mathrm{~g}_{3} \\
\mathrm{~g}_{2}+i \mathrm{~g}_{3} & \mathrm{~g}_{0}-\mathrm{g}_{1}
\end{array}\right)
\end{aligned}
$$

where parameters $\left\{g_{0}, g_{1}, g_{2}, g_{3}\right\}$ are called the Stokes parameters, and the vector

$$
\mathbf{g}=\left(\begin{array}{c}
\mathrm{g}_{0} \\
\mathrm{~g}_{1} \\
\mathrm{~g}_{2} \\
\mathrm{~g}_{3}
\end{array}\right)=\left(\begin{array}{c}
\left\langle\left|E_{H}\right|^{2}+\left|E_{V}\right|^{2}\right\rangle \\
\left\langle\left|E_{H}\right|^{2}-\left|E_{V}\right|^{2}\right\rangle \\
2 \Re\left\langle E_{H} E_{V}^{*}\right\rangle \\
-2 \Im\left\langle E_{H} E_{V}^{*}\right\rangle
\end{array}\right)
$$

is called the Stokes vector. In these expressions, $E$ is the complex electric field received in the subscripted polarization, $\langle\cdot\rangle$ denotes ensemble averaging (multilooking in the SAR context), and $\Re$ and $\Im$ denote the real and imaginary parts of the complex field, respectively. Note that the transmit polarization is not included in the subscripting. The transmit polarization for a traditional dual-pol radar is either $\mathrm{H}$ or $\mathrm{V}$, for the hybrid (CL-pol) mode is either left or right circular ( $\mathrm{L}$ and $\mathrm{R}$ ), and for the $\pi / 4$ compact mode is $\mathrm{H}+\mathrm{V}$ oriented at $45^{\circ}\left(L_{45^{\circ}}\right)$.

As aforementioned, the state of polarization of an electromagnetic wave can be characterized by the DoP, which is defined in terms of the covariance matrix elements as [17]

$$
\mathcal{P}=\left(1-4 \frac{|\boldsymbol{\Gamma}|}{(\operatorname{tr} \boldsymbol{\Gamma})^{2}}\right)^{\frac{1}{2}}=\left(1-\frac{4\left[a_{1} a_{2}-\left(a_{3}^{2}+a_{4}^{2}\right)\right]}{\left(a_{1}+a_{2}\right)^{2}}\right)^{\frac{1}{2}}
$$


where $|\boldsymbol{\Gamma}|$ and $\operatorname{tr} \boldsymbol{\Gamma}$ are the determinant and trace of $\boldsymbol{\Gamma}$, respectively. We see that (6) is invariant under unitary transformations (such as rotation); thus, the DoP does not depend on the particular orthogonal pair of polarimetric channels chosen to measure the backscattered wave [26]. Hence, the DoP is invariant of the (receiver) polarization basis. Note that the wave is totally depolarized for $\mathcal{P}=0$, totally polarized for $\mathcal{P}=1$, and partially polarized when $\mathcal{P} \in] 0,1[$. The estimation of the DoP from expression (6) can be conducted by estimating the parameters of the covariance matrix, i.e., $a_{i}, i=1, \ldots, 4$. For this purpose, we introduce a modified Stokes vector $\tilde{\mathbf{g}}$ defined as

$$
\tilde{\mathrm{g}}=\frac{1}{2}\left(\mathrm{~g}_{0}+\mathrm{g}_{1}, \mathrm{~g}_{0}-\mathrm{g}_{1}, \mathrm{~g}_{0}+\mathrm{g}_{2}, \mathrm{~g}_{0}+\mathrm{g}_{3}\right)^{T} .
$$

Throughout this paper, we refer to the four elements of $\tilde{\mathrm{g}}$ as four intensity images. We also consider that for each intensity image, $q$ looks are taken. Note that full polarimetric radar systems measure the complex-valued elements of the scattering matrix $\boldsymbol{S}$ (also called the Sinclair matrix [27]) rather than the modified Stokes parameters. The scattering matrix $S$ relates the electric vector $\boldsymbol{E}^{r}$ of the received (or backscattered) field to the transmitted (or incident) illumination $\boldsymbol{E}^{t}$ by

$$
\boldsymbol{E}^{r}=\boldsymbol{S} \boldsymbol{E}^{t}, \boldsymbol{S}=\left(\begin{array}{cc}
S_{\mathrm{HH}} & S_{\mathrm{HV}} \\
S_{\mathrm{VH}} & S_{\mathrm{VV}}
\end{array}\right) .
$$

However, in what follows, we show that the modified Stokes parameters introduced in this paper are of great mathematical interest (in addition to their physical relevance in optical polarimetric imagery [28, pp. 340-341]) and can be employed to derive simple estimators of the DoP.

\section{THEORY}

\section{A. Joint Distribution of Multilook SAR Intensity Images}

SAR data are usually multilook processed for speckle reduction. A multilook image is obtained by averaging multiple independent measurements. In order to estimate the DoP, we first study the statistical properties of the multilook intensity vector $\tilde{\mathrm{g}}$. For this purpose, we derive the Laplace transform of $\tilde{\mathrm{g}}$. It is well known that, under the usual assumption of fully developed speckle, the Jones vector $\boldsymbol{E}$ is distributed according to a complex circular Gaussian distribution [18] whose probability density function (pdf) is

$$
p_{G}(\boldsymbol{E})=\frac{1}{\pi^{2}|\boldsymbol{\Gamma}|} \exp \left(-\boldsymbol{E}^{\dagger} \boldsymbol{\Gamma}^{-1} \boldsymbol{E}\right) .
$$

Considering $q$-independent (1-look) samples $\boldsymbol{E}_{j}$, where $j=$ $1, \ldots, q$ from such a distribution, the $q$-look Hermitian covariance matrix $\bar{\Gamma}$ is expressed as

$$
\overline{\boldsymbol{\Gamma}}=\frac{1}{q} \sum_{j=1}^{q} \boldsymbol{E}_{j} \boldsymbol{E}_{j}^{\dagger} .
$$

Let $\mathbf{A}_{\boldsymbol{E}}=q \overline{\boldsymbol{\Gamma}}$. Based on Appendix A, the matrix $\mathbf{A}_{\boldsymbol{E}}$ is distributed according to a Wishart distribution whose Laplace transform is

$$
L_{\mathbf{A}_{\boldsymbol{E}}}(\boldsymbol{\Theta})=\mathrm{E}\left[\exp \left(-\operatorname{tr}\left(\boldsymbol{\Theta}^{T} \mathbf{A}_{\boldsymbol{E}}\right)\right)\right]=\left|\mathbb{I}_{2}+\boldsymbol{\Gamma} \boldsymbol{\Theta}\right|^{-q}
$$

where $\mathbb{I}_{2}$ is a $2 \times 2$ identity matrix, $\Theta$ is a $2 \times 2$ complex Hermitian matrix, and $|\cdot|$ represents the determinant. The random Hermitian matrix $\mathbf{A}_{E}$ is expressed in terms of the $q$-look Stokes parameters as follows:

$$
\mathbf{A}_{\boldsymbol{E}}=\sum_{j=1}^{q}\left(\begin{array}{cc}
\left|E_{H_{j}}\right|^{2} & E_{H_{j}} E_{V_{j}}^{*} \\
E_{V_{j}} E_{H_{j}}^{*} & \left|E_{V_{j}}\right|^{2}
\end{array}\right)=\frac{q}{2}\left(\begin{array}{cc}
\mathrm{g}_{0}+\mathrm{g}_{1} & \mathrm{~g}_{2}-i \mathrm{~g}_{3} \\
\mathrm{~g}_{2}+i \mathrm{~g}_{3} & \mathrm{~g}_{0}-\mathrm{g}_{1}
\end{array}\right) .
$$

By definition, the Laplace transform of the pdf of $\tilde{\mathrm{g}}$ is $L_{\tilde{\mathrm{g}}}(\boldsymbol{\theta})=$ $\mathrm{E}\left[\exp \left(-\boldsymbol{\theta}^{T} \tilde{\mathbf{g}}\right)\right]$, with $\boldsymbol{\theta}=\left(\theta_{1}, \theta_{2}, \theta_{3}, \theta_{4}\right)^{T}$. Therefore, using (7) and (12), one can obtain

$$
\begin{aligned}
L_{\tilde{\mathbf{g}}}(\boldsymbol{\Theta}) & =\mathrm{E}\left[\exp \left(-\operatorname{tr}\left(\boldsymbol{\Theta}^{T} \mathbf{A}_{\boldsymbol{E}}\right)\right)\right] \\
& =L_{\mathbf{A}_{\boldsymbol{E}}}(\boldsymbol{\Theta})
\end{aligned}
$$

where

$$
\boldsymbol{\Theta}=\left(\begin{array}{cc}
\Theta_{1} & \Theta_{3}+i \Theta_{4} \\
\Theta_{3}-i \Theta_{4} & \Theta_{2}
\end{array}\right)
$$

with $\quad q \Theta_{1}=\theta_{1}+\left(\theta_{3}+\theta_{4}\right) / 2, \quad q \Theta_{2}=\theta_{2}+\left(\theta_{3}+\theta_{4}\right) / 2$, $q \Theta_{3}=\theta_{3} / 2$, and $q \Theta_{4}=\theta_{4} / 2$. Hence, using (11) and (13), and after calculating the determinant, we obtain the Laplace transform of the pdf of the modified Stokes vector $\tilde{\mathbf{g}}$ as

$$
\begin{aligned}
L_{\tilde{\mathbf{g}}}(\boldsymbol{\theta}) & =\frac{1}{\widetilde{P}(\boldsymbol{\theta})^{q}} \\
\widetilde{P}(\boldsymbol{\theta}) & =1+\boldsymbol{\alpha}^{T} \boldsymbol{\theta}+\beta\left[2 \theta_{1} \theta_{2}+\theta_{3} \theta_{4}+\left(\theta_{1}+\theta_{2}\right)\left(\theta_{3}+\theta_{4}\right)\right] \\
\boldsymbol{\alpha} & =\frac{1}{q}\left(a_{1}, a_{2}, \frac{a_{1}+a_{2}}{2}+a_{3}, \frac{a_{1}+a_{2}}{2}+a_{4}\right)^{T} \\
\beta & =\frac{1}{2 q^{2}}\left(a_{1} a_{2}-a_{3}^{2}-a_{4}^{2}\right) .
\end{aligned}
$$

We see that $\widetilde{P}(\boldsymbol{\theta})$ is a quadratic affine polynomial; thus, the distribution of the $q$-look intensity vector $\tilde{\mathrm{g}}$ is a multivariate gamma distribution (MGD) with $d=4$ (see Appendix A for more details). We note that $d \in \mathbb{N}$ is the dimension of the gamma distribution, i.e., the number of available intensity images. Moreover, based on the characteristics of an MGD, the marginal distributions of two $(d=2)$ or three $(d=3)$ multilook intensity images are also MGDs. Thus, one can set the corresponding $\theta_{i}$ to zero in order to obtain the related polynomials leading to the marginal pdfs. The parameter $q$ is both the number of looks and the shape parameter of the gamma distribution. The earlier results are valid for any $q>0$; thus, $q$ can be chosen as either the number of looks (with values in the set of positive integers $\mathbb{N}$ ) or the equivalent number of looks [9], [29] (with values in the set of real positive numbers $\mathbb{R}^{+}$).

\section{B. Marginal Distribution of Two Multilook Intensity Images}

In the general case, where the four intensity images (defined by $\tilde{\mathrm{g}}$ ) are available, the properties of an MGD can be used to directly derive the estimators of the DoP [30] under different polarizations and incidence angles [31]. However, in dual-pol SAR systems where only two intensity images are delivered, 
estimation of the DoP is challenging. In the particular case of two multilook intensity images $(d=2)$, it can be shown that $\left(\tilde{\mathrm{g}}_{0}, \tilde{\mathrm{g}}_{1}\right)^{T}$ follows a BGD whose pdf is (see Appendix A):

$$
\begin{aligned}
p_{\mathrm{BGD}}\left(\widetilde{\mathrm{g}}_{0}, \widetilde{\mathrm{g}}_{1}\right)=\exp \left(-\frac{a_{2} \widetilde{\mathrm{g}}_{0}+a_{1} \widetilde{\mathrm{g}}_{1}}{2 q \beta}\right) \frac{\widetilde{\mathrm{g}}_{0}^{q-1} \widetilde{\mathrm{g}}_{1}^{q-1}}{(2 \beta)^{q} \Gamma(q)} \\
\times f_{q}\left(c \widetilde{\mathrm{g}}_{0} \widetilde{\mathrm{g}}_{1}\right) \mathbb{I}_{\mathbb{R}_{+}^{2}}\left(\widetilde{\mathrm{g}}_{0}, \widetilde{\mathrm{g}}_{1}\right)
\end{aligned}
$$

where $\beta=1 / 2 q^{2}\left(a_{1} a_{2}-r\right), c=q^{2} r /\left(a_{1} a_{2}-r\right)^{2}, r=a_{3}^{2}+$ $a_{4}^{2}, \mathbb{I}_{\mathbb{R}^{2}}\left(\tilde{\mathrm{g}}_{0}, \tilde{\mathrm{g}}_{1}\right)$ is the indicator function on $\mathbb{R}^{+} \times \mathbb{R}^{+}$, and $f_{q}(z)=\sum_{j=0}^{\infty} z^{j} /(\Gamma(q+j) j !)$ is related to confluent hypergeometric and modified Bessel functions [32, p. 374]. We see that this distribution is parameterized by $a_{1}, a_{2}$, and $r$. Hence, we can derive the ML estimators of these three parameters. Moreover, based on the first- and second-order moments of such a distribution (given in Appendix A), the moment estimators of $a_{1}, a_{2}$, and $r$ can also be derived.

The earlier distributions, which are derived for $q \in \mathbb{R}^{+}$and $d \in\{1,2,3,4\}$, generalize those of [20, eq. (16)] derived for $q=1$ and $d=2$, [19, eq. (30)] derived for $q \in \mathbb{N}$ and $d=2$, and that of Chatelain et al. [30] derived for $q=1$ and $d \in$ $\{1,2,3,4\}$. It is worth noting that while the introduced modified Stokes vector $\tilde{\mathbf{g}}$ follows an MGD, the Stokes vector $\mathbf{g}$ is not distributed according to an MGD (since $L_{\mathbf{g}}(\boldsymbol{\theta})=1 / P(\boldsymbol{\theta})^{q}$, where the polynomial $P(\boldsymbol{\theta})$ is not affine). ${ }^{1}$ The latter makes clear that the modified Stokes vector $\tilde{\mathrm{g}}$ is more appropriate than the standard Stokes vector $\mathrm{g}$ for deriving the estimators of the DoP. In the following sections, we derive ML and MoM estimators of $\left(a_{1}, a_{2}, r\right)$ using the properties of a BGD. These estimators lead to the ML and MoM estimators of the DoP based on two intensity images.

\section{ML Estimators}

The ML method can be applied in the bivariate case $(d=$ 2) since a closed-form expression of the density is available, as shown in (16). Considering $n$-independent vectors $\tilde{\mathbf{g}}[1], \ldots, \tilde{\mathbf{g}}[n]$ from such a distribution and differentiating the joint density of $\tilde{\mathbf{g}}_{1: n}=(\tilde{\mathbf{g}}[1], \ldots, \tilde{\mathbf{g}}[n])^{T}$, which is denoted as $p\left(\tilde{\mathbf{g}}_{1: n}\right)$, with respect to $a_{1}$ and $a_{2}$, and solving the equations $\partial p\left(\tilde{\mathbf{g}}_{1: n}\right) / \partial a_{1}=\partial p\left(\tilde{\mathbf{g}}_{1: n}\right) / \partial a_{2}=0$, we obtain the ML estimators of $a_{1}$ and $a_{2}$ as

$$
\widehat{a}_{l_{\mathrm{ML}}}=\frac{1}{n} \sum_{j=1}^{n} \widetilde{\mathrm{g}}_{l}[j], \quad l=1,2
$$

where $n$ is the number of pixels used for the estimation. In practice, $\widehat{a}_{l_{\mathrm{ML}}}$ is calculated for each pixel of the multilook intensity image by using a sliding square window (centered on the considered pixel) and computing the empirical mean over the $n$ pixels contained in the window. The parameter $r$ is the correlation coefficient for the BGD; thus, it is upper bounded by 1 . Moreover, we have $r=a_{3}^{2}+a_{4}^{2}$, which makes $r$ to be positive; thus, we have $r \in[0,1]$. By replacing $a_{1}$ and $a_{2}$ in $\partial p\left(\tilde{\mathbf{g}}_{1: n}\right) / \partial r=0$ by their ML estimators, one can show that

\footnotetext{
${ }^{1}$ The distribution of the Stokes vector is more complex; however, it can be obtained from the distribution of $\tilde{\mathbf{g}}$ by a change of variables.
}

the ML estimators of $r$, which is denoted as $\widehat{r}_{\mathrm{ML}}$, satisfies the following nonlinear relation:

$$
\widehat{a}_{1_{\mathrm{ML}}} \widehat{a}_{2_{\mathrm{ML}}}-\widehat{r}_{\mathrm{ML}}-\frac{q}{n} \sum_{j=1}^{n} \widetilde{\mathrm{g}}_{0}[j] \widetilde{\mathrm{g}}_{1}[j] \frac{f_{q+1}\left(\widehat{c} \widetilde{\mathrm{g}}_{0}[j] \widetilde{\mathrm{g}}_{1}[j]\right)}{f_{q}\left(\widehat{c} \widetilde{\mathrm{g}}_{0}[j] \widetilde{\mathrm{g}}_{1}[j]\right)}=0 .
$$

We note that (18) also ensures that the ML estimators of $a_{1}$ and $a_{2}$ decouple from one another and $r$. The practical determination of $\widehat{r}_{\mathrm{ML}}$ is achieved by using a Newton-Raphson procedure under the constraint $\widehat{r}_{\mathrm{ML}} \in[0,1]$. The ML estimators of $a_{1}, a_{2}$, and $r$ are then plugged into (6), yielding the DoP ML estimator based on two multilook polarimetric images

$$
\widehat{\mathcal{P}}_{\mathrm{ML}}=\left[1-\frac{4\left(\widehat{a}_{1_{\mathrm{ML}}} \widehat{a}_{2_{\mathrm{ML}}}-\widehat{r}_{\mathrm{ML}}\right)}{\left(\widehat{a}_{1_{\mathrm{ML}}}+\widehat{a}_{2_{\mathrm{ML}}}\right)^{2}}\right]^{\frac{1}{2}} .
$$

\section{MoM Estimators}

The first- and second-order moments of a BGD are given in Appendix A. Considering (16) along with these moments, one can find the first- and second-order moments of $\left(\tilde{\mathrm{g}}_{0}, \tilde{\mathrm{g}}_{1}\right)^{T}$ as

$$
\begin{aligned}
m_{1} & =\mathrm{E}\left[\widetilde{\mathrm{g}}_{0}\right]=a_{1}, \quad m_{2}=\mathrm{E}\left[\widetilde{\mathrm{g}}_{1}\right]=a_{2} \\
m_{12} & =\mathrm{E}\left[\widetilde{\mathrm{g}}_{0} \widetilde{\mathrm{g}}_{1}\right]=\frac{r}{q}+a_{1} a_{2} .
\end{aligned}
$$

Hence, moment estimators of $a_{1}, a_{2}$, and $r$ can be obtained as follows:

$$
\begin{aligned}
\widehat{a}_{l_{\mathrm{MoM}}} & =\widehat{m}_{l}=\frac{1}{n} \sum_{j=1}^{n} \widetilde{\mathrm{g}}_{l}[j] \quad l=1,2 \\
\widehat{r}_{\mathrm{MoM}} & =q\left(\widehat{m}_{12}-\widehat{m}_{1} \widehat{m}_{2}\right) \\
& =\frac{q}{n} \sum_{j=1}^{n} \widetilde{\mathrm{g}}_{0}[j] \widetilde{\mathrm{g}}_{1}[j]-q \widehat{a}_{1_{\mathrm{MoM}}} \widehat{a}_{2_{\mathrm{MoM}}} .
\end{aligned}
$$

These estimators are then plugged into (6), yielding the MoM estimator of the DoP, which is denoted as $\widehat{\mathcal{P}}_{\mathrm{MoM}}$. We see that the moment estimators of $a_{1}, a_{2}$ are the same as their ML estimators. On the other hand, the ML estimator of $r$ incorporates a weighted second-order moment compared with its moment estimator $\widehat{r}_{\text {MoM. }}$. We note that these results generalize those in [33] derived for $q=1$ (using a different method) in an optical polarimetric imagery context.

\section{Data And Study Sites}

The performance of the proposed DoP estimators are evaluated on both synthetic and real data. In this paper, we use a set of synthetic polarimetric images as well as four real data sets, which are acquired by RADARSAT-2 spaceborne and NASA/ JPL AirSAR systems. The full-pol data sets are used to generate hybrid/compact and linear dual-pol data. We compare the DoP estimators in different SAR modes over various terrain types such as urban, vegetation, and ocean. The synthetic and real data sets are described in what follows. 
TABLE I

DoP And The Corresponding Covariance Matrices of Synthetic Polarimetric Images

\begin{tabular}{|c||c|c|c|c|c|c|c|c|c|c|}
\hline & $\Gamma_{0}$ & $\Gamma_{1}$ & $\Gamma_{2}$ & $\Gamma_{3}$ & $\Gamma_{4}$ & $\Gamma_{5}$ & $\Gamma_{6}$ & $\Gamma_{7}$ & $\Gamma_{8}$ & $\Gamma_{9}$ \\
\hline \hline$a_{1}$ & 2 & 5 & 15 & 1 & 16 & 82 & 18 & 30 & 2 & 1.25 \\
\hline$a_{2}$ & 2 & 5 & 6 & 1 & 3.6 & 17 & 11 & 14 & 2 & 26 \\
\hline$a_{3}$ & 0 & 1 & 0.2 & 0.4 & 0 & 0 & 7 & 16 & 0.6 & 0 \\
\hline$a_{4}$ & 0 & 0 & 0.5 & $\sqrt{0.14}$ & 0 & 13 & 8 & 8 & 1.8 & 5.5 \\
\hline \hline $\mathcal{P}$ & 0 & $\mathbf{0 . 2 0}$ & $\mathbf{0 . 4 5}$ & $\mathbf{0 . 5 5}$ & $\mathbf{0 . 6 3}$ & $\mathbf{0 . 7 0}$ & $\mathbf{0 . 7 7}$ & $\mathbf{0 . 9 0}$ & $\mathbf{0 . 9 5}$ & $\mathbf{0 . 9 9}$ \\
\hline
\end{tabular}
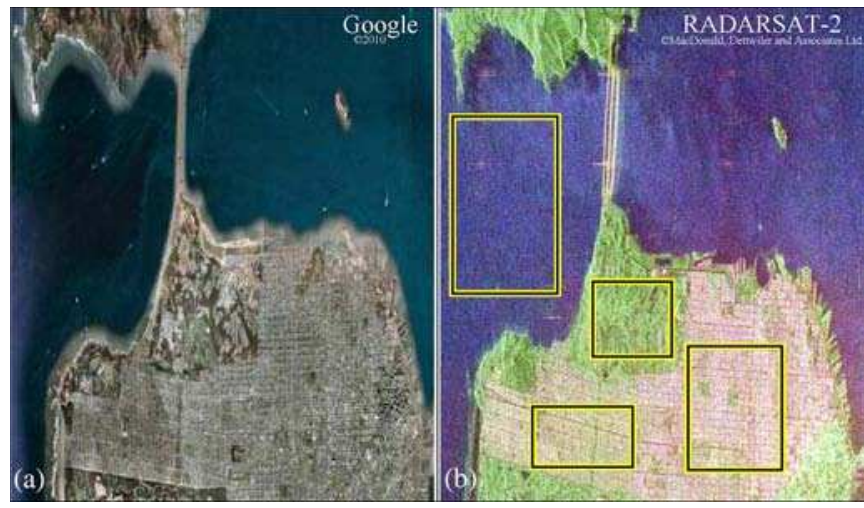

Fig. 1. San Francisco Bay, CA. USA. (a) Google Earth image of the area. (b) Pauli RGB image of the RADARSAT-2 fully polarimetric data set (Red, $\left|S_{\mathrm{HH}}-S_{\mathrm{VV}}\right|$; Green, $\left|S_{\mathrm{HV}}+S_{\mathrm{VH}}\right|$; Blue, $\left.\mid S_{\mathrm{HH}}+S_{\mathrm{VV}}\right)$. The four outlined areas in (b) are (from left to right) ocean, urban 1, park, and urban 2 regions. The original image has a size of $1270 \times 1450$ pixels.

\section{A. Synthetic Data}

To evaluate the performance of the proposed estimators on synthetic data, we consider ten different values of $\boldsymbol{\Gamma}$ denoted as $\boldsymbol{\Gamma}_{0}, \boldsymbol{\Gamma}_{1}, \ldots, \boldsymbol{\Gamma}_{9}$, as shown in Table I. The synthetic images were generated from these covariance matrices, without specifically considering any particular polarization (see [30] for more details).

\section{B. Real Data}

1) Full Polarimetic Data: We evaluate and compare the proposed estimators using two C-band data sets acquired by RADARSAT-2, as well as two L-band data sets acquired by the NASA/JPL AirSAR system. RADARSAT-2 is a Canadian C-band SAR satellite launched in December 2007. It provides many operating modes, including linear dual-pol and quad-pol modes, and supports right- and left-look imaging. The NASA/ JPL AirSAR system is a side-looking three-frequency airborne polarimetric system. It became operational in 1988, simultaneously providing $\mathrm{P}-, \mathrm{L}-$, and $\mathrm{C}-$ band fully polarimetric data.

The RADARSAT-2 data sets are acquired in fine quad-pol mode over San Francisco Bay, CA. USA $\left(+37^{\circ} 45^{\prime} 0^{\prime \prime} \mathrm{N}\right.$, $\left.+122^{\circ} 17^{\prime} 0^{\prime \prime} \mathrm{W}\right)$, and over Vancouver, BC, Canada $\left(+49^{\circ} 15^{\prime} 0^{\prime \prime} \mathrm{N},+123^{\circ} 6^{\prime} 0^{\prime \prime} \mathrm{W}\right)$. The Google Earth and Pauli RGB images of these data sets are shown in Figs. 1 and 2 , respectively. These data sets consist of three main regions: man-made structures (urban areas, bridges, etc.), water areas (ocean, seas, and lakes), and vegetation areas.

The NASA/JPL AirSAR data sets are acquired in quadpol mode over Flevoland, The Netherlands $\left(+52^{\circ} 20^{\prime} 00^{\prime \prime} \mathrm{N}\right.$, $+5^{\circ} 23^{\prime} 00^{\prime \prime}$ E), and over San Francisco Bay, CA. USA. The
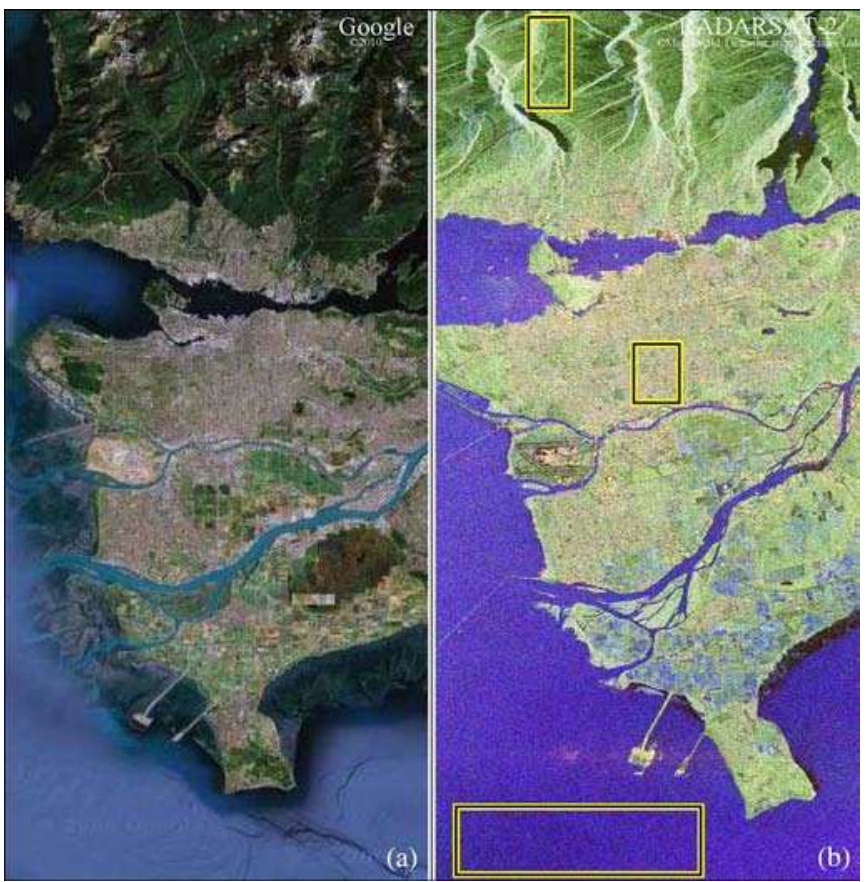

Fig. 2. Vancouver, BC, Canada. (a) Google Earth image of the area. (b) Pauli RGB image of the RADARSAT-2 fully polarimetric data set (Red, $\mid S_{\mathrm{HH}}-$ $S_{\mathrm{VV}} \mid$; Green, $\left|S_{\mathrm{HV}}+S_{\mathrm{VH}}\right|$; Blue, $\left.\left|S_{\mathrm{HH}}+S_{\mathrm{VV}}\right|\right)$. The outlined areas in (b) are (from top to bottom) vegetation, urban, and sea regions. The original image has a size of $1985 \times 11393$ pixels.

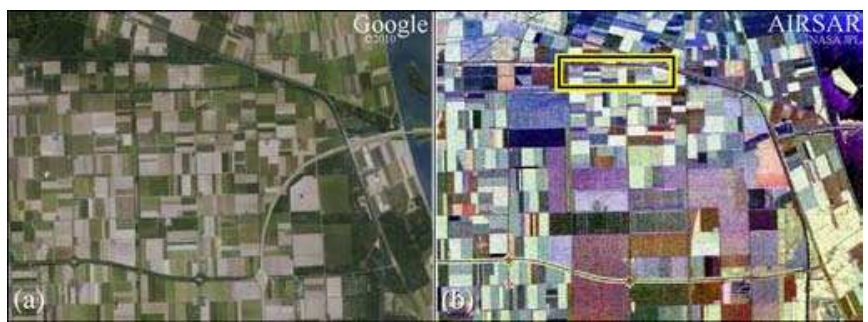

Fig. 3. Flevoland, The Netherlands. (a) Google Earth image of the area (b) Pauli RGB image of the NASA/JPL AirSAR fully polarimetric data set (Red, $\left|S_{\mathrm{HH}}-S_{\mathrm{VV}}\right|$; Green, $\left|S_{\mathrm{HV}}+S_{\mathrm{VH}}\right|$; Blue, $\left|S_{\mathrm{HH}}+S_{\mathrm{VV}}\right|$ ). The outlined area in (b) is a test area in which a number of high-voltage transmission towers are present. The original image has a size of $1024 \times 750$ pixels.

Flevoland data set covers a large agricultural area of horizontally flat topography and homogeneous soils, some man-made structures, and a small water area. The San Francisco data set covers nearly the same regions as the first RADARSAT- 2 data set. The Google Earth and Pauli RGB images of these data sets are shown in Figs. 3 and 4, respectively.

We use the scattering vector $\vec{k}=\left(S_{\mathrm{HH}}, S_{\mathrm{HV}}, S_{\mathrm{VH}}, S_{\mathrm{VV}}\right)^{T}$ to represent full polarimetric SAR data. Scattering vectors in 


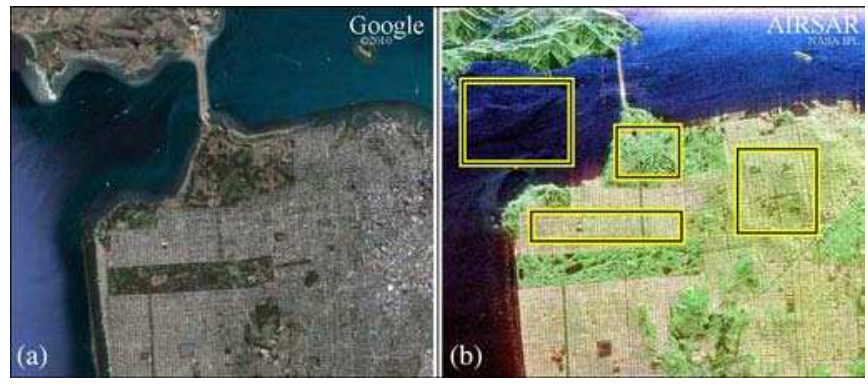

Fig. 4. San Francisco Bay, CA. USA. (a) Google Earth image of the area. (b) Pauli RGB image of the NASA/JPL AirSAR fully polarimetric data set (Red, $\left|S_{\mathrm{HH}}-S_{\mathrm{VV}}\right|$; Green, $\left|S_{\mathrm{HV}}+S_{\mathrm{VH}}\right|$; Blue, $\left.\left|S_{\mathrm{HH}}+S_{\mathrm{VV}}\right|\right)$. The four outlined areas in (b) are (from left to right) ocean, urban 1, park, and urban 2 regions. The original image has a size of $1024 \times 900$ pixels.

hybrid/compact and linear dual-pol modes are built from quadpol data as follows (see Appendix B for more details).

2) Dual-Pol Data: The scattering vectors for traditional dual-pol modes are given by

$$
\begin{aligned}
& \vec{k}_{\mathrm{DP} 1}=\left(S_{\mathrm{HH}}, S_{\mathrm{HV}}\right)^{T} \\
& \vec{k}_{\mathrm{DP} 2}=\left(S_{\mathrm{VH}}, S_{\mathrm{VV}}\right)^{T} \\
& \vec{k}_{\mathrm{DP} 3}=\left(S_{\mathrm{HH}}, S_{\mathrm{VV}}\right)^{T} .
\end{aligned}
$$

3) Hybrid/Compact Data: The scattering vectors for the $\pi / 4$ [10], DCP with right circular transmit, [11], and right circular transmit, linear receive (CL-pol) [12] modes are given by

$$
\begin{gathered}
\vec{k}_{\pi / 4}=\frac{1}{\sqrt{2}}\left(S_{\mathrm{HH}}+S_{\mathrm{HV}}, S_{\mathrm{VV}}+S_{\mathrm{VH}}\right)^{T} \\
\vec{k}_{\mathrm{DCP}}=\frac{1}{2}\left(S_{\mathrm{HH}}-S_{\mathrm{VV}}-i\left[S_{\mathrm{HV}}+S_{\mathrm{VH}}\right], S_{\mathrm{HH}}\right. \\
\left.\quad+S_{\mathrm{VV}}+S_{\mathrm{HV}}-S_{\mathrm{VH}}\right)^{T} \\
\vec{k}_{\mathrm{CL}-\mathrm{pol}}=\frac{1}{\sqrt{2}}\left(S_{\mathrm{HH}}-i S_{\mathrm{HV}},-i S_{\mathrm{VV}}+S_{\mathrm{VH}}\right)^{T} .
\end{gathered}
$$

We note that under the scattering reciprocity and in the backscatter alignment convention, we have $S_{\mathrm{HV}}=S_{\mathrm{VH}}$ [34].

\section{EXPERIMENTAL RESUlts AND DisCUSSION}

\section{A. Performance Analysis With Synthetic Data}

The performance of the ML and MoM estimators are compared using the synthetic data presented in Section IV-A. Fig. 5 shows the log MSEs of the DoP estimates obtained with two images using the ML (red diamond markers) and MoM (blue square markers) estimators for different numbers of looks. Comparing these MSEs with those corresponding to four images (black circle markers), the performance loss that occurs when using only two polarimetric images, instead of four, can be clearly observed (note that the ML and the MoM estimations coincide when four images are observed). Moreover, both ML and MoM estimators deliver good estimations when $\mathcal{P}$ is close to 1 , whereas both estimators tend to estimate the DoP less accurately when $\mathcal{P}$ is close to 0 . This confirms that the estimation of the DoP is more precise for highly polarized regions compared with moderately polarized areas. Moreover, we see

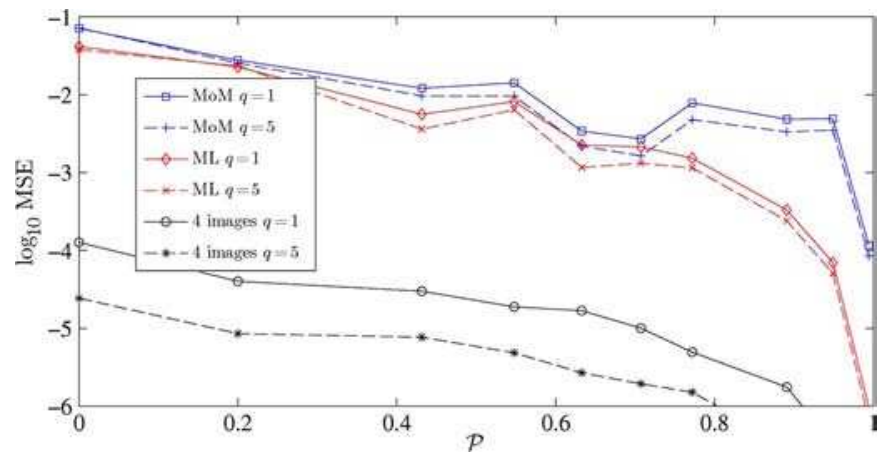

Fig. 5. $\log$ MSE of $\widehat{\mathcal{P}}$ as a function of $\mathcal{P}$ for polarization matrices $\boldsymbol{\Gamma}_{i}$ and $n=11 \times 11$. Number of Monte Carlo realizations is $10^{4}$, and $q=$ number of looks.

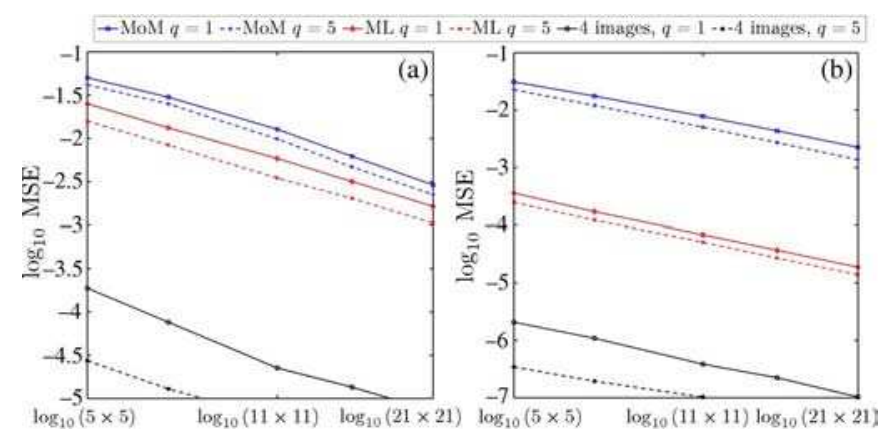

Fig. 6. (a) $\log$ MSE of $\widehat{\mathcal{P}}$ versus the logarithm of the sample size for the matrix $\boldsymbol{\Gamma}_{2}$. (b) Same as (a) for the matrix $\boldsymbol{\Gamma}_{8}$. Number of Monte Carlo realizations is $10^{4}$, and $q=$ number of looks.

that the ML estimators of the DoP deliver better estimations compared with the MoM estimators (which is a classical result). Fig. 6 shows the performance of the different estimators as a function of the sample size $n$ for different numbers of looks and two matrices $\boldsymbol{\Gamma}_{2}$ and $\boldsymbol{\Gamma}_{8}$ (given in Table I). The usual linear relation between $\log _{10} \mathrm{MSE}$ and $\log _{10}(n)$ is observed in both single- and multilook cases. These figures also show the gain of performance obtained with the ML method compared with the MoM. We note that ML is significantly better than MoM for $\Gamma_{8}(\mathcal{P}$ close to 1$)$. In other words, the DoP estimation using the ML method is more precise than MoM, particularly in highly polarized regions (which is explained by the weighted secondorder moment of $\widehat{r}_{\mathrm{ML}}$ ). As aforementioned, speckle noise is better reduced with a larger number of looks $q$, which in turn leads to a better estimation performance. We note that, in Fig. 6, the larger the number of looks $q$, the better the estimation performance is for both ML and MoM estimators.

\section{B. Performance Analysis in Dual-Pol SAR Imagery}

The performance of the ML and MoM estimators are compared using dual-polarized SAR data presented in Section IV-B2. Maps of the DoP in (HH, HV) and (HV,VV) dual-pol modes for RADARSAT-2 San Francisco Bay and AirSAR Flevoland data sets are presented in Figs. 7 and 8. A visual inspection of the results suggests that all the estimators have a similar global behavior with the water areas having the maximum DoP and the vegetation areas having the minimum DoP. Since the DoP estimation based on four images is the 


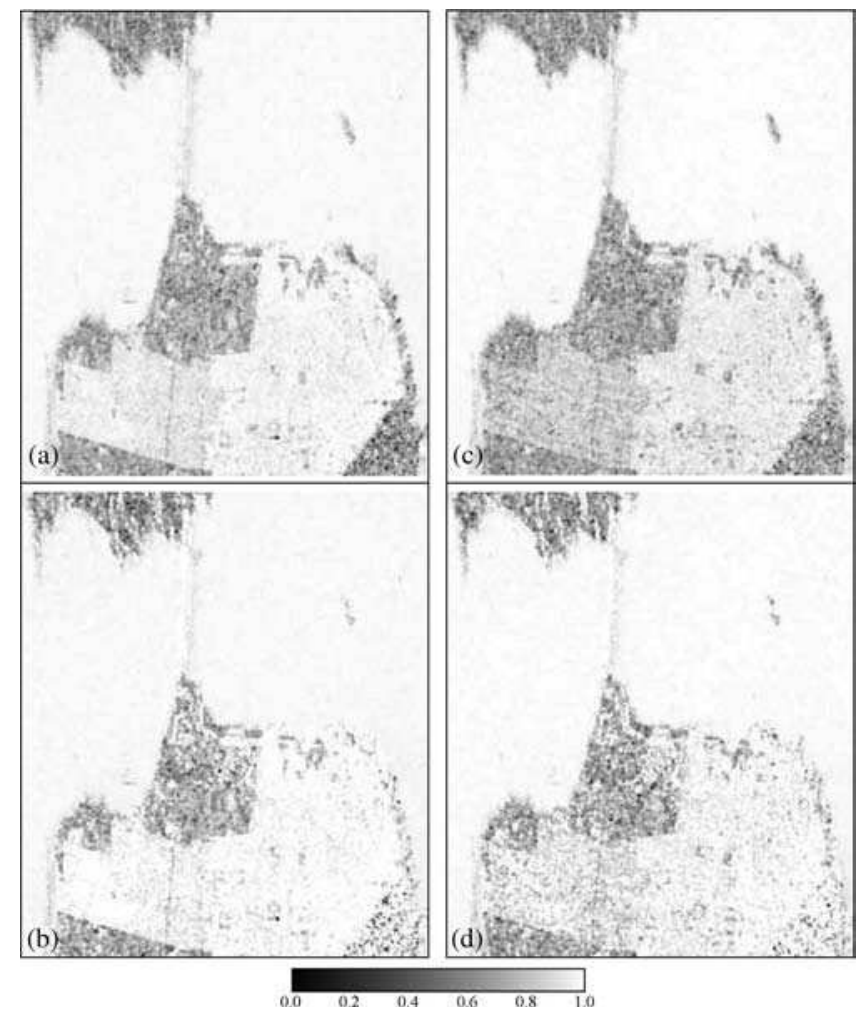

Fig. 7. Comparison of the DoP ML and MoM estimators in dual-pol modes. RADARSAT-2 image of San Francisco Bay, CA. (a) $\widehat{\mathcal{P}}_{\mathrm{ML}}$ in (HH, HV) mode. (b) $\widehat{\mathcal{P}}_{\mathrm{MoM}}$ in (HH, HV) mode. (c) $\widehat{\mathcal{P}}_{\mathrm{ML}}$ in $(\mathrm{VH}, \mathrm{VV})$ mode. (d) $\widehat{\mathcal{P}}_{\mathrm{MoM}}$ in $(\mathrm{VH}$, VV) mode. A sliding window covering $n=9 \times 9$ pixels is used.

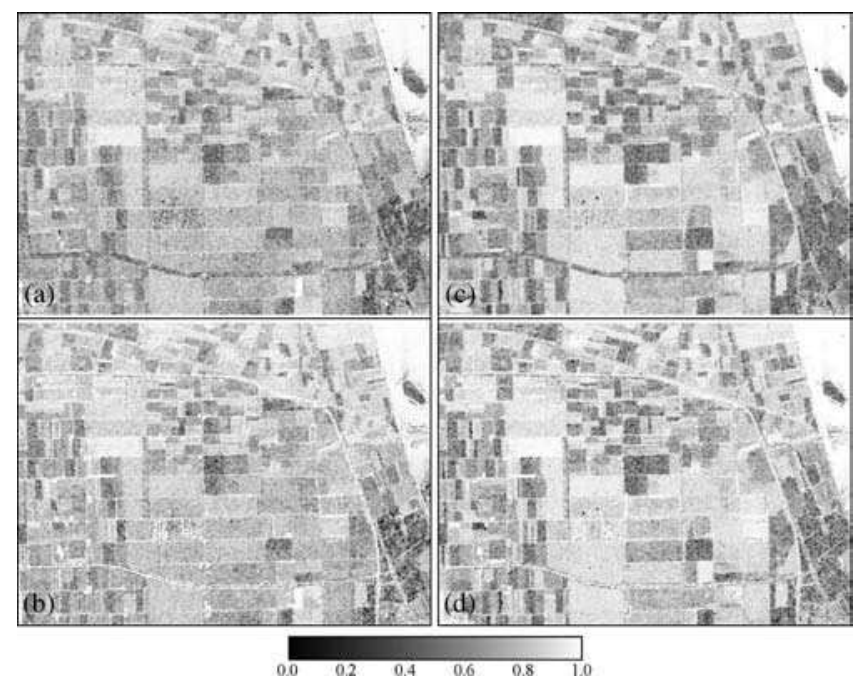

Fig. 8. Comparison of the DoP ML and MoM estimators in dual-pol modes. NASA/JPL AirSAR image of Flevoland, The Netherlands. (a) $\widehat{\mathcal{P}}_{\mathrm{ML}}$ in $(\mathrm{HH}$, HV) mode. (b) $\widehat{\mathcal{P}}_{\mathrm{MoM}}$ in (HH, HV) mode. (c) $\widehat{\mathcal{P}}_{\mathrm{ML}}$ in $(\mathrm{VH}, \mathrm{VV})$ mode. (d) $\widehat{\mathcal{P}}_{\mathrm{MoM}}$ in $(\mathrm{VH}, \mathrm{VV})$ mode. A sliding window covering $n=5 \times 5$ pixels is used.

benchmark (classical) estimation, we consider it as the reference in this paper. We use scatter plots in order to examine how well the DoP estimations in dual-pol modes fit the DoP estimations based on four images.

Fig. 9 shows the scatter plots of the DoP ML and MoM estimations over different regions of the San Francisco Bay area in (HH, HV) dual-pol mode. The scatter plots have been normalized in the range of $[0,1]$. The closer the estimated values are to the diagonal line, the better the estimation, compared with the given reference (note that the scatter plots of the ocean region are shown in a different scale). The DoP reference maps, computed using four images, are shown in Fig. 10. There are several phenomena to note in Fig. 9. The ML estimators of the DoP deliver better estimations over all the different terrain types, which are present in our data set, compared with MoM estimators. Both ML and MoM estimators tend to overestimate the DoP for urban areas (values over the diagonal line). These estimators deliver good estimations for values of $\widehat{\mathcal{P}}$ close to 1 (ML over the ocean being the best). However, they tend to estimate the DoP less accurately in the vegetation areas with $\widehat{\mathcal{P}}$ values close to 0.7 (the MoM estimator over the park area being the worst). Scatter plots derived from other presented data sets suggest the same results. This is in agreement with the results from synthetic data presented in Fig. 5. Fig. 11 shows the scatter plots of the ML and MoM estimators for different sizes of the sliding window over the park region. It confirms that a bigger sliding window leads to better estimation results for both ML and MoM estimators. We also notice that for each sliding window, the ML method gives estimators with smaller variances (i.e., better performance), compared with the corresponding MoM estimators.

\section{Comparison of DoP Estimations in Hybrid/Compact and Linear Dual-Pol Modes}

In this section, we study and compare the ML estimators of the DoP, based on two intensity images, in hybrid/compact and linear dual-pol modes. As aforementioned, the phase information simplifies the estimators of the DoP and can also provide us with potentially useful information (see [31] for more details). However, the analysis conducted in this paper mainly applies to applications where the phase information is not available. Hybrid/compact dual-pol data are simulated using (25) based on quad-pol data. Figs. 12 and 13 show the maps of the DoP obtained using two intensity images delivered in each hybrid/compact and linear dual-pol mode, respectively, for RADARSAT-2 San Francisco Bay and AirSAR Flevoland data sets. We note that Figs. 12 and 13(a) and (b) are the same as Figs. 7 and 8(a) and (c), respectively. A visual inspection of the results for the RADARSAT-2 San Francisco Bay image suggests that the DoP estimates in traditional linear dual-pol modes [see Figs. 12(a) and (b)] are better suited for the segmentation of the image, compared with other hybrid/compact dualpol modes. The mean $\mu$ and variance $\sigma^{2}$ of the estimated DoP in each polarimetric mode over four test regions, shown in Fig. 1, are given in Table II. We note that the proposed MLE estimators are asymptotically unbiased (i.e., considering a large number of samples); thus, the variance can demonstrate the performance of the estimators. Since the ocean region is a homogeneous region, the estimates with small variances demonstrate better estimation performance. We notice that, in Table II, traditional linear dual-pol modes, i.e., (HH, HV) and (VH, VV), give estimators with smaller variances (i.e., better performance) compared with other dual-pol modes. We highlight that the 

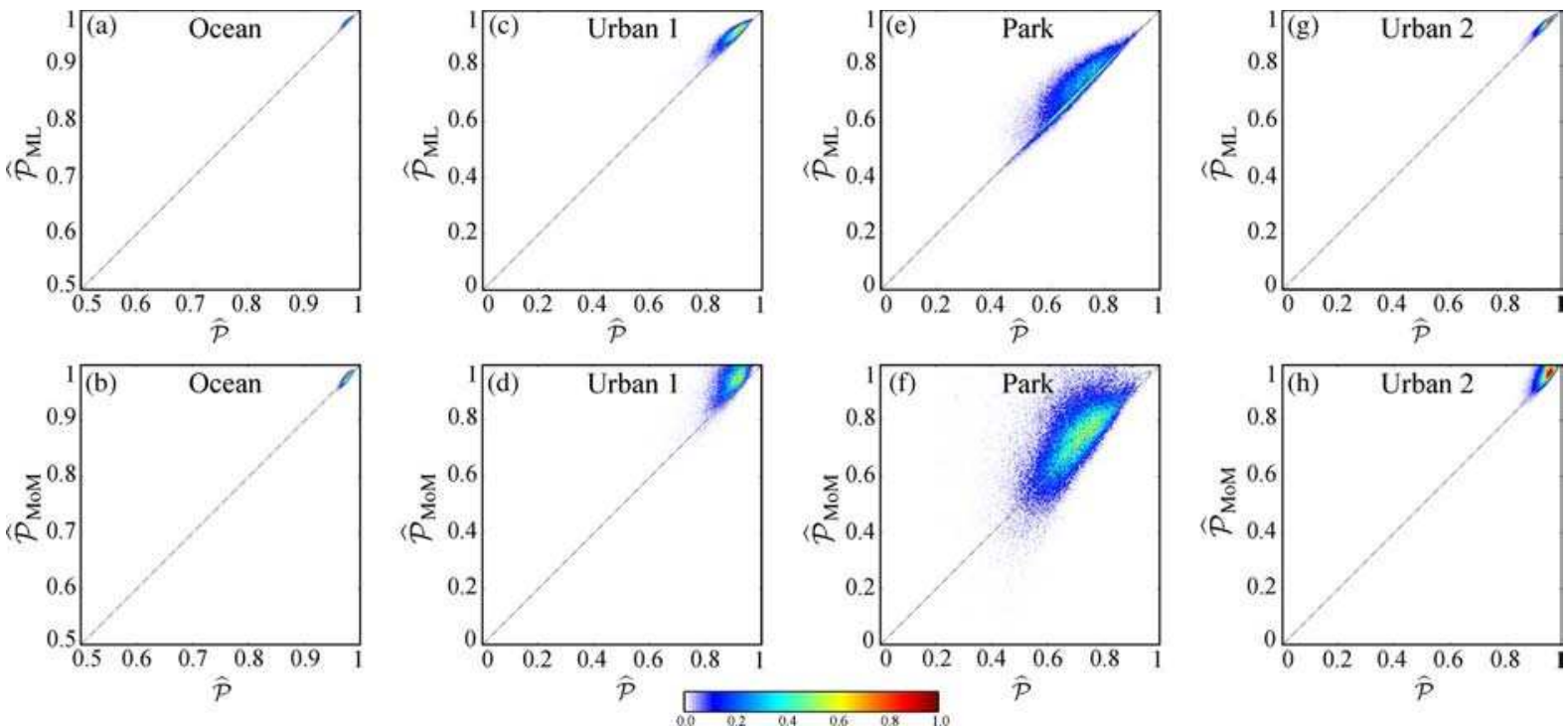

Fig. 9. Scatter plots of the DoP ML and MoM estimates in (HH, HV) dual-pol mode versus the DoP estimates based on four intensity images over different regions of the San Francisco Bay image (RADARSAT-2 data set). On the abscissa are $\widehat{\mathcal{P}}$ values based on four intensity images, and on the ordinate are the estimated values in the dual-pol mode. Perfectly estimated values lie along the diagonal line. (a) $\widehat{\mathcal{P}}_{\text {ML }}$ over ocean. (b) $\widehat{\mathcal{P}}_{\text {MoM }}$ over ocean. (c) $\widehat{\mathcal{P}}_{\text {ML }}$ over urban 1 .

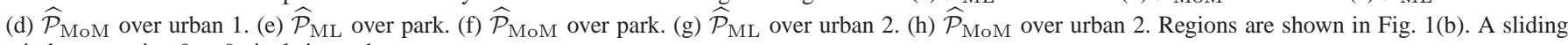
window covering $9 \times 9$ pixels is used.

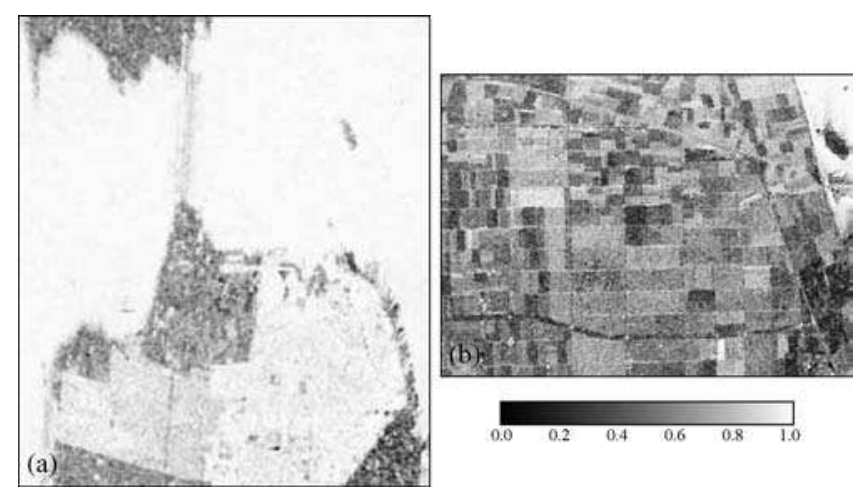

Fig. 10. Maps of the DoP based on four intensity images. (a) San Francisco Bay, CA. USA, $n=9 \times 9$. (b) Flevoland, The Netherlands, $n=5 \times 5$.

latter conclusion is coherent with previous studies, although using a different approach (see, e.g., [35, Table III]).

The statistics of the DoP ML estimators in different hybrid/ compact and linear dual-pol modes are further studied for water, urban, and vegetation areas in Figs. 14, 15, and 16, respectively. For each of such areas, three test regions are chosen from the RADARSAT-2 Vancouver, the RADARSAT-2 San Francisco Bay, and the AirSAR San Francisco Bay data sets. The test regions are outlined in Figs. 1, 2, and 4. We note that the selected water regions are homogeneous regions with oddbounce scattering mechanism. On the other hand, the urban areas include buildings, streets, grass, trees, and other structures. Therefore, the urban regions represent combinations of different scattering mechanisms, although even-bounce scattering is dominant. The vegetation regions exhibit a volume scattering mechanism. In these figures, the variance of the DoP shows that urban and vegetation areas are more random than ocean areas (which is well known). Moreover, we notice that the mean of the DoP is different for these areas; thus, mean values can
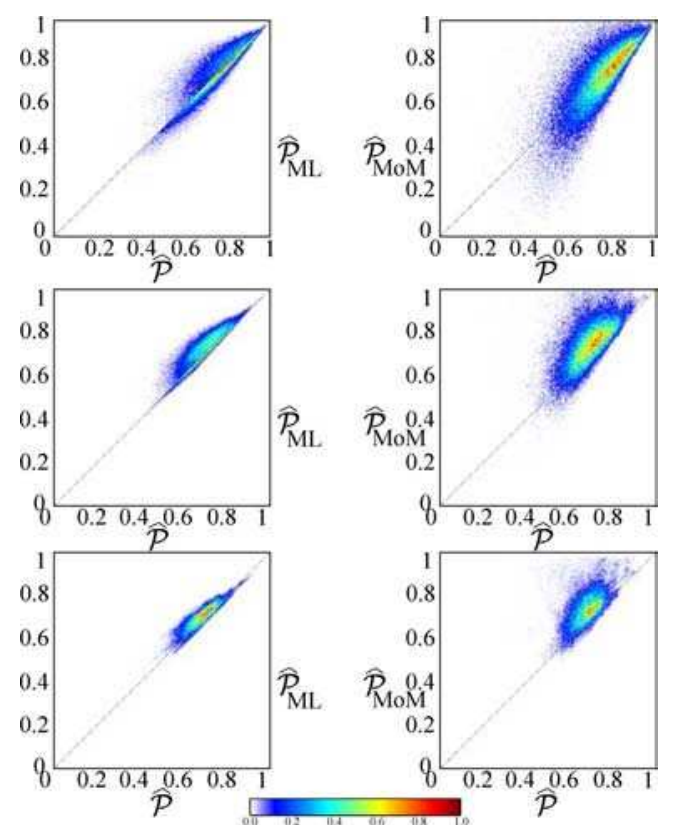

Fig. 11. Comparison of the scatter plots of the DoP ML and MoM estimates for different sliding windows over the park region. On the abscissa are $\widehat{\mathcal{P}}$ based on four intensity images, and on the ordinate are the estimated values in the (HH, HV) dual-pol mode. Perfectly estimated values lie along the diagonal line. (Left column) $\widehat{\mathcal{P}}_{\mathrm{ML}}$. (Right column) $\widehat{\mathcal{P}}_{\mathrm{MoM}}$. (First row) $n=5 \times 5$. (Second row) $n=11 \times 11$. (Third row) $n=19 \times 19$.

provide discriminating information for different regions. These results are of interest for the classification and segmentation of polarimetric SAR images, in particular, the distinction of urban and vegetation areas from water areas (compare Figs. 15 and 16 to Fig. 14). We note that, due to the high level of detail involved in the urban environment, the full benefit of the DoP for urban applications is achieved by performing the DoP estimation on high-resolution SAR images. This is an interesting subject for 

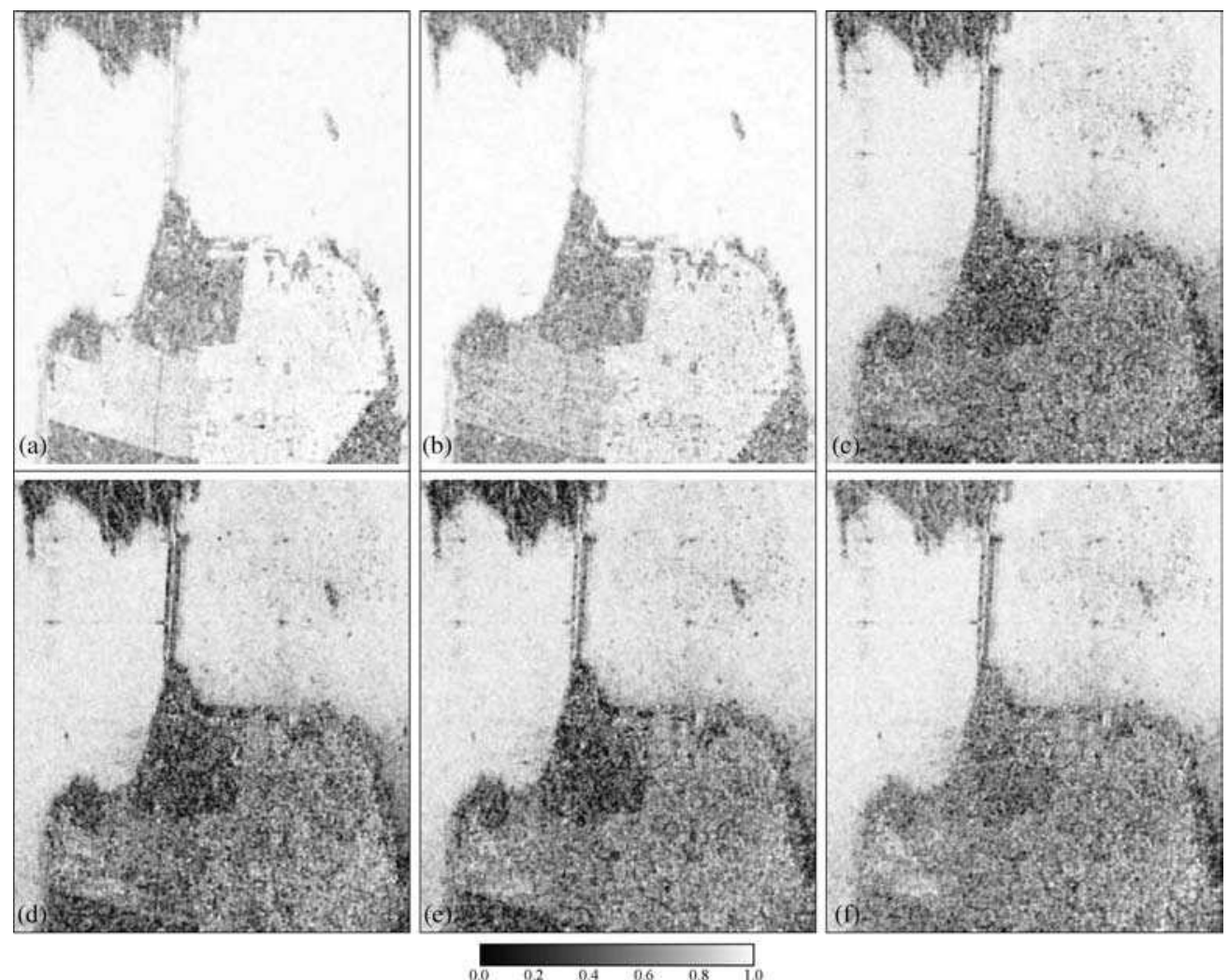

Fig. 12. Maps of the DoP (ML estimates) in different hybrid/compact and linear dual-pol modes, over San Francisco Bay area (RADARSAT-2 data set). (a) HH-HV. (b) VH-VV. (c) HH-VV (d) DCP. (e) CL-pol. (f) $\pi / 4$. A sliding window covering $9 \times 9$ pixels is used.
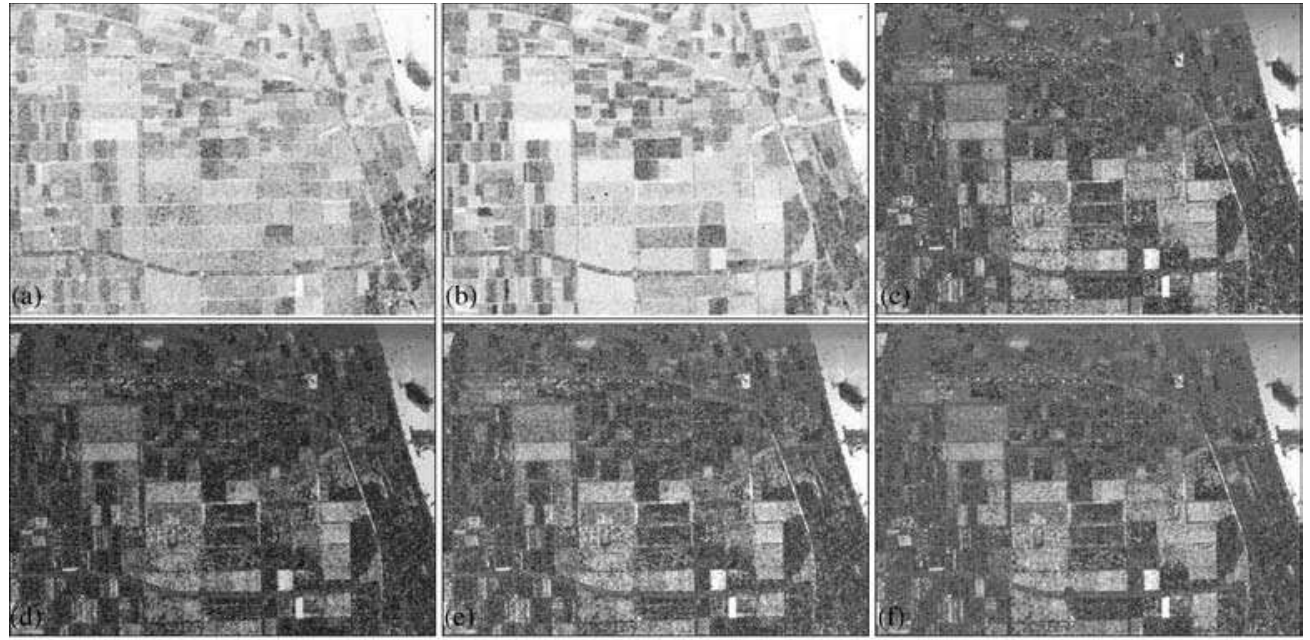

Fig. 13. Maps of the DoP (ML estimates) in different hybrid/compact and linear dual-pol modes, over Flevoland, The Netherlands (AirSAR data set). (a) HH-HV. (b) VH-VV. (c) HH-VV (d) DCP. (e) CL-pol. (f) $\pi / 4$. A sliding window covering $5 \times 5$ pixels is used.

future work, notably with the advent of new emerging highresolution SAR systems such as RADARSAT-2 ultrafine mode $(3 \mathrm{~m})$, and TerraSAR-X high-resolution Spotlight mode (up to $1 \mathrm{~m}$ ) imagery. As the last part of our analysis, we examine the capability of DoP ML estimations in hybrid/compact and linear dual-pol modes to detect man-made metallic objects in a natural environment. For this purpose, we study a subset of the AirSAR Flevoland image (see Fig. 3) containing high-tension electrical transmission lines in an agricultural environment. In this case, the targets are high-voltage transmission towers, and the clutter background is the backscatter from the agricultural area. The Google Earth and Pauli RGB images of this test region are shown in Fig. 17(a) and (b), respectively. The eight high-voltage transmission towers are manually identified on the Google Earth image of the area (see the red boxes). In the Pauli image, the pink areas show the important dihedral contribution from high-voltage transmission towers.Some of these towers are bright enough to be identified on the Pauli RGB image, whereas others are hidden in the agricultural environment. We highlight that the detection of the transmission towers 
TABLE II

Mean $\mu$ And Variance $\sigma^{2}$ of the DoP ML Estimates in Different Polarimetric Modes, Over Ocean, Park, Urban 1, And Urban 2 Regions of RADARSAT-2 San Francisco Bay Data Set (Regions Are Shown in Fig. 1)

\begin{tabular}{|c|c|c|c|c|c|c|c|c|c|c|c|c|}
\hline & \multicolumn{2}{|c|}{ HH-HV } & \multicolumn{2}{|c|}{ VH-VV } & \multicolumn{2}{|c|}{ HH-VV } & \multicolumn{2}{|c|}{ DCP } & \multicolumn{2}{|c|}{ CL-pol } & \multicolumn{2}{|c|}{$\pi / 4$} \\
\hline & $\mu$ & $\sigma^{2}$ & $\mu$ & $\sigma^{2}$ & $\mu$ & $\sigma^{2}$ & $\mu$ & $\sigma^{2}$ & $\mu$ & $\sigma^{2}$ & $\mu$ & $\sigma^{2}$ \\
\hline Ocean & $0.975^{1}$ & $1 e-4$ & 0.982 & $\begin{array}{l}1 e-4 \\
1\end{array}$ & 0.929 & $12 \mathrm{e}-4$ & 0.910 & $16 e-4$ & 0.910 & $17 e-4$ & 0.928 & $12 e-4$ \\
\hline Park & 0.721 & $111 \mathrm{e}-4$ & 0.692 & i111e-4 & 0.466 & $316 e-4$ & 0.398 & & 0.405 & $344 e-4$ & 0.537 & $255 \mathrm{e}-4$ \\
\hline Urban 1 & 0.920 & $20 \mathrm{e}-4$ & 0.827 & $50 e-4$ & 0.603 & $198 \mathrm{e}-4$ & 0.562 & $285 e-4$ & 0.604 & :213e-4 & 0.647 & $177 \mathrm{e}-4$ \\
\hline Urban 2 & 0.949 & $16 e-4$ & 0.913 & $30 e-4$ & 0.602 & $221 e-4$ & 0.584 & & 0.603 & & 0.618 & -4 \\
\hline
\end{tabular}

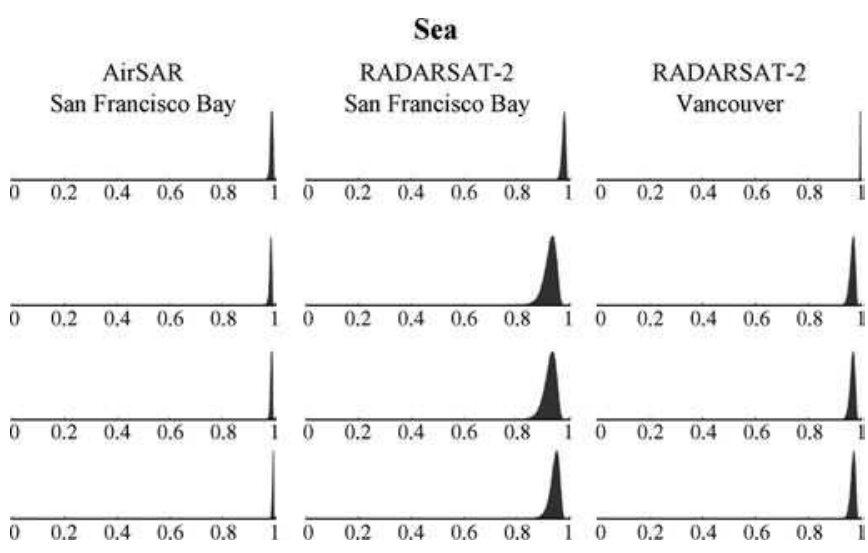

Fig. 14. Histograms of DoP ML estimates in different polarimetric modes over three different water areas. (First row) HH-HV. (Second row) DCP. (Third row) CL-pol. (Fourth row) $\pi / 4$. (Left column) Water area from the AirSAR San Francisco Bay data. (Middle column) Water area from the RADARSAT-2 San Francisco Bay data. (Right column) Water area from the RADARSAT-2 Vancouver data. Regions are shown in Figs. 1, 2, and 4. A sliding window covering $9 \times 9$ pixels is used.

\section{Urban}

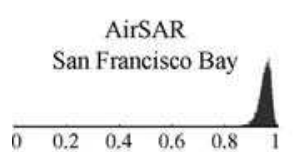

\section{RADARSAT-2} San Francisco Bay
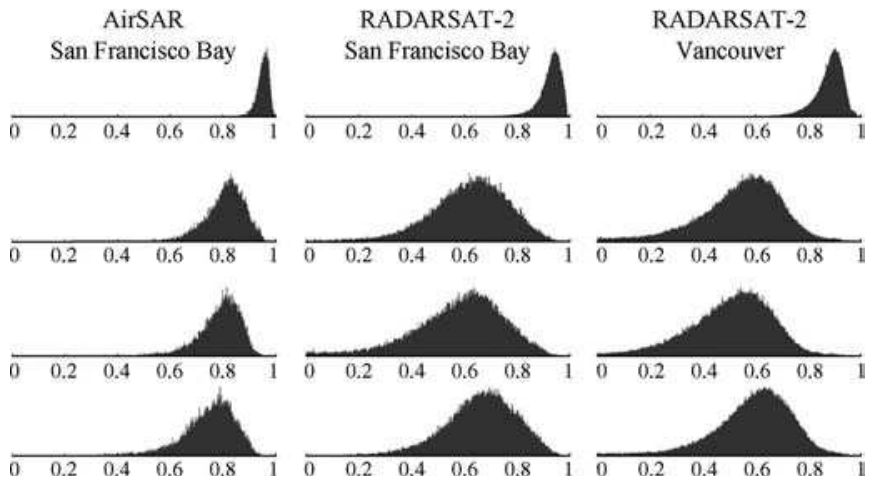

Fig. 15. Histograms of DoP ML estimates in different polarimetric modes over three different urban areas. (First row) HH-HV. (Second row) DCP. (Third row) CL-pol. (Fourth row) $\pi / 4$. (Left column) Urban area from the AirSAR San Francisco Bay data. (Middle column) Urban area from the RADARSAT-2 San Francisco Bay data. (Right column) Urban area from the RADARSAT-2 Vancouver data. Regions are shown in Figs. 1, 2, and 4. A sliding window covering $9 \times 9$ pixels is used.

differs from the previous classification context; in particular, transmission towers introduce a preferred direction and thus may be more visible in some dual-pol modes than the others because of their alignment. Fig. 17(e) and (f) shows that all of
Park

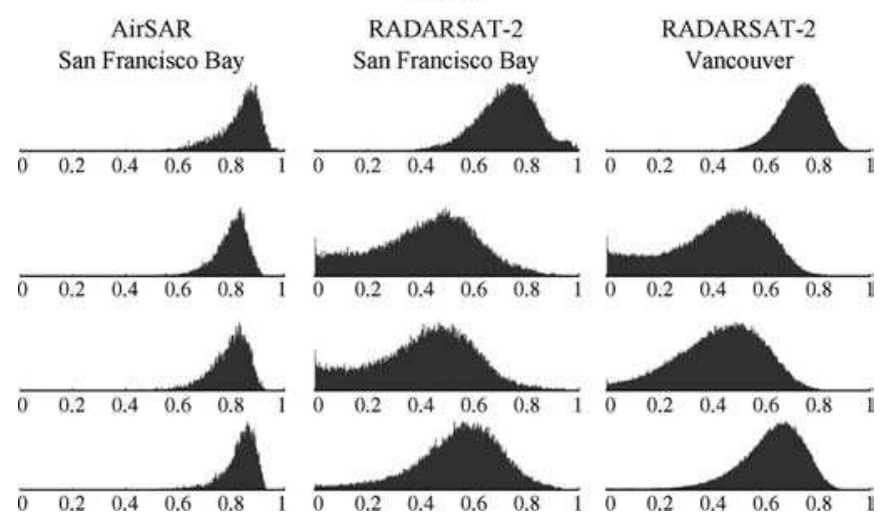

Fig. 16. Histograms of DoP ML estimates in different polarimetric modes over three different vegetation areas. (First row) HH-HV. (Second row) DCP. (Third row) CL-pol. (Fourth row) $\pi / 4$. (Left column) Vegetation area from the AirSAR San Francisco Bay data. (Middle column) Vegetation area from the RADARSAT-2 San Francisco Bay data. (Right column) Vegetation area from the RADARSAT-2 Vancouver data. Regions are shown in Figs. 1, 2, and 4. A sliding window covering $9 \times 9$ pixels is used.

the eight towers are clearly detected by DoP ML estimations in CL-pol and $\pi / 4$ modes, whereas the traditional dual-pol modes in Fig. 17(c) and (d) demonstrate lower target detection performance in this test case. We note that, in Fig. 17(c)-(f), a threshold has been manually chosen so that a maximum number of targets is visible. We see that the DoP is a good parameter to separate deterministic objects from a random environment in partial polarimetry. These results are of great interest in a variety of applications dealing with the detection of targets in a natural environment, such as maritime surveillance, vessel detection, ship observation, and so forth [31].

\section{CONCLUSION}

The joint distribution of multilook polarimetric SAR intensity images was derived and expressed in closed form. ML and MoM estimators of the DoP, based on hybrid/compact and linear dual-pol SAR intensity images, were proposed. The performance of these estimators was then evaluated over synthetic and real multilook polarimetric SAR data, acquired by RADARSAT-2 spaceborne and NASA/JPL AirSAR systems. Experimental results suggest that ML estimators outperform MoM estimators over different terrain types, such as urban, 


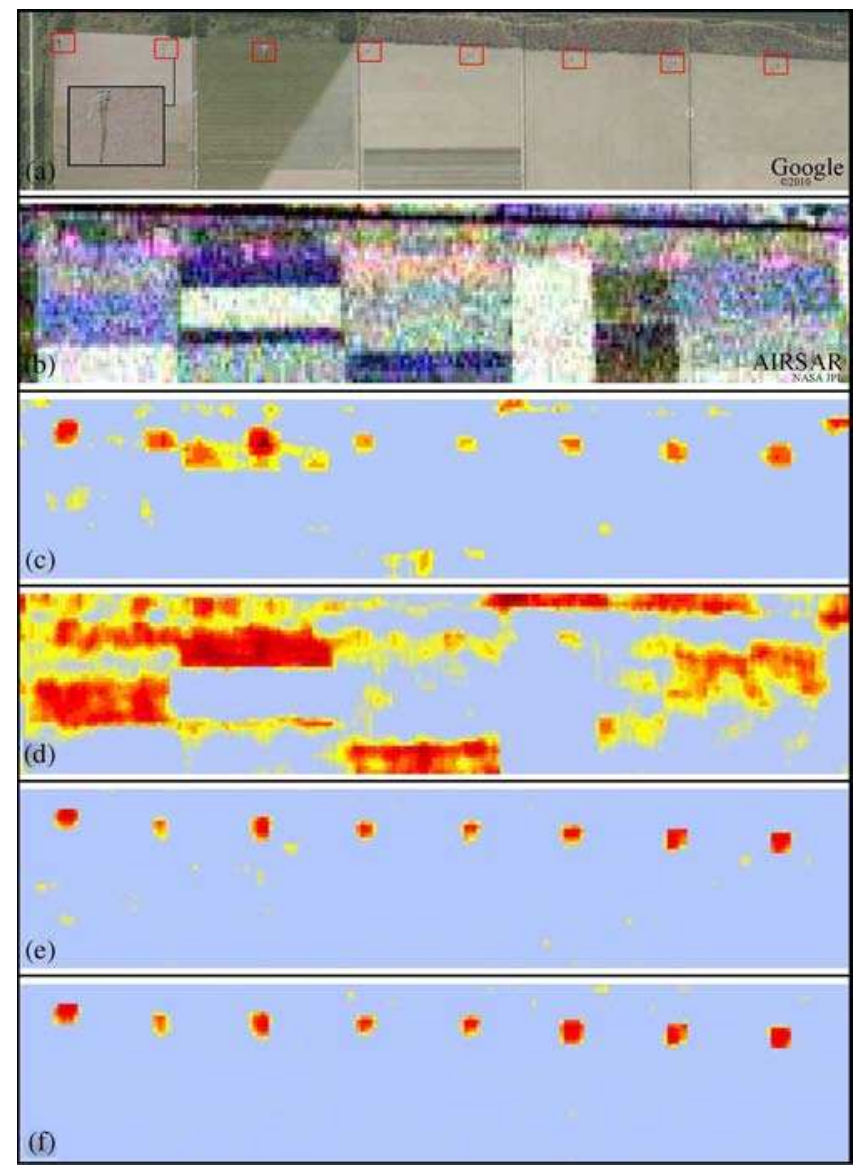

Fig. 17. Man-made metallic object detection based on the DoP ML estimations. (a) Google Earth image of the test area in which eight high-voltage transmission towers (red boxes) are present. (b) Pauli RGB subimage of the area derived from the AirSAR Flevoland fully polarimetric data set (Red, $\left|S_{\mathrm{HH}}-S_{\mathrm{VV}}\right|$; Green, $\left|S_{\mathrm{HV}}+S_{\mathrm{VH}}\right|$; Blue, $\left.\left|S_{\mathrm{HH}}+S_{\mathrm{VV}}\right|\right)$. (c) DoP ML estimation in (HH, HV) mode. (d) DoP ML estimation in (VH, VV) mode. (e) DoP ML estimation in CL-pol mode. (f) DoP ML estimation in $\pi / 4$ mode. A sliding window covering $5 \times 5$ pixels is used.

vegetation, and ocean. The estimators of the DoP, based on two intensity images, deliver better performance in ocean and urban regions compared with vegetation areas. These estimators suggest that DoP estimates in traditional linear dual-pol modes, i.e., $(\mathrm{HH}, \mathrm{HV})$ and $(\mathrm{VH}, \mathrm{VV})$, better distinguish different regions, compared with other modes. However, it is shown that the DoP is robust in hybrid/compact dual-pol modes (i.e., CL-pol and $\pi / 4$ ) for detecting man-made metallic objects in a natural environment. The statistics derived in this paper should prove useful in the study of hybrid/compact and linear dual- and quad-pol SAR polarimetry. The developed DoP estimators are of interest in different applications of hybrid/compact and linear dual-pol SAR data, such as image segmentation and object detection.

\section{APPENDiX A \\ Theoretical Probability Distributions}

\section{Complex Wishart Distribution}

Let us consider $\boldsymbol{\xi}$ as a zero-mean $p$-variate circular complex Gaussian random vector. The pdf of $\boldsymbol{\xi}$ is given by

$$
p_{G}(\boldsymbol{\xi})=\frac{1}{\pi^{p}\left|\boldsymbol{\Sigma}_{\boldsymbol{\xi}}\right|} \exp \left(-\boldsymbol{\xi}^{\dagger} \boldsymbol{\Sigma}_{\boldsymbol{\xi}}^{-1} \boldsymbol{\xi}\right)
$$

where $\Sigma_{\boldsymbol{\xi}}$ is the covariance matrix, and $|\cdot|$ represents the determinant. If $\boldsymbol{\xi}_{1}, \boldsymbol{\xi}_{2}, \ldots, \boldsymbol{\xi}_{q}$ is a sample of $q$-independent complex-valued vectors from such a distribution, then the sample Hermitian covariance matrix

$$
\hat{\mathbf{\Sigma}}_{\boldsymbol{\xi}}=\frac{1}{q} \sum_{j=1}^{q} \boldsymbol{\xi}_{j} \boldsymbol{\xi}_{j}^{\dagger}
$$

is the ML estimator of $\boldsymbol{\Sigma}_{\boldsymbol{\xi}}$ [36]. Consider $\mathbf{A}_{\boldsymbol{\xi}}=q \hat{\boldsymbol{\Sigma}}_{\boldsymbol{\xi}}$. The joint distribution of the elements of the matrix $\mathbf{A}_{\boldsymbol{\xi}}$ is called a complex Wishart distribution whose pdf is expressed as [36, Th. 5.1]

$$
p_{\mathrm{W}}\left(\mathbf{A}_{\boldsymbol{\xi}}\right)=\frac{\left|\mathbf{A}_{\boldsymbol{\xi}}\right|^{q-p}}{B\left(\boldsymbol{\Sigma}_{\boldsymbol{\xi}}\right)} \exp \left[-\operatorname{tr} \boldsymbol{\Sigma}_{\boldsymbol{\xi}}^{-1} \mathbf{A}_{\boldsymbol{\xi}}\right]
$$

where $B\left(\boldsymbol{\Sigma}_{\boldsymbol{\xi}}\right)=\pi^{1 / 2 p(p-1)} \Gamma(q) \cdots \Gamma(q-p+1)\left|\boldsymbol{\Sigma}_{\boldsymbol{\xi}}\right|^{q}$. The Laplace transform of such a distribution is expressed as $L_{\mathbf{A}_{\boldsymbol{\xi}}}(\boldsymbol{\Theta})=\mathrm{E}\left[\exp \left(-\operatorname{tr}\left(\boldsymbol{\Theta}^{T} \mathbf{A}_{\boldsymbol{\xi}}\right)\right)\right]=\left|\mathbb{I}_{p}+\boldsymbol{\Sigma}_{\boldsymbol{\xi}} \boldsymbol{\Theta}\right|^{-q}$, where $\mathbb{I}_{p}$ is the $p \times p$ identity matrix, and $\boldsymbol{\Theta}$ is a $p \times p$ complex Hermitian matrix such that $L_{\mathbf{A}_{\xi}}(\Theta)<\infty$.

$M G D$ : In the literature, MGDs have several nonequivalent definitions. In this paper, we consider the definitions provided in [37]. Let $d \in \mathbb{N}$ be the set of positive integers. The probability distribution $\mu$ on $\mathbb{R}_{+}^{d}$, called an MGD, which is denoted by $\mathrm{Ga}(q, P)$, is defined by its Laplace transform as [37]

$$
L_{\boldsymbol{\mu}}(\mathbf{z})=[P(\mathbf{z})]^{-q}
$$

where the shape parameter satisfies $q>0$, and the scale parameter $P(\boldsymbol{z})$ is an affine polynomial (i.e., $\forall j: \partial^{2} P / \partial z_{j}^{2}=0$ ) with the constant term equal to 1 . Note that $d$ is the dimension of the gamma distribution, i.e., the number of available intensity images in this paper. Moreover, not all affine polynomials give rise to a valid Laplace transform. In this paper, we focus on a particular case of MGD with a quadratic affine polynomial, i.e.,

$$
P(\boldsymbol{z})=1+\sum_{i=1}^{d} p_{i} z_{i}+\sum_{1 \leq i<j \leq d} p_{i j} z_{i} z_{j} .
$$

This family of polynomials and the necessary and sufficient conditions under which they give rise to a valid pdf have been studied in detail in [37] and [38]. In particular, based on (30) and for $d=2$, a bivariate gamma distribution (BGD) is obtained with $P(\boldsymbol{z})=1+p_{1} z_{1}+p_{2} z_{2}+p_{12} z_{1} z_{2}$, where $p_{1}$, $p_{2}>0$, and $0<p_{12} \leq p_{1} p_{2}$. The pdf of a random vector $\boldsymbol{x}=$ $\left(x_{1}, x_{2}\right)^{T}$ following such a BGD is given by [39]

$$
\begin{aligned}
p_{\mathrm{BGD}}(\boldsymbol{x})=\exp \left(-\frac{p_{2} x_{1}+p_{1} x_{2}}{p_{12}}\right) & \frac{x_{1}^{q-1} x_{2}^{q-1}}{p_{12}^{q} \Gamma(q)} \\
& \times f_{q}\left(c x_{1} x_{2}\right) \mathbb{I}_{\mathbb{R}_{+}^{2}}(\boldsymbol{x})
\end{aligned}
$$

where $\quad c=\left(p_{1} p_{2}-p_{12}\right) / p_{12}^{2}, \quad$ and $\quad f_{q}(z)=\sum_{j=0}^{\infty} z^{j} /$ $(\Gamma(q+j) j !)$ is related to confluent hypergeometric and modified Bessel functions [32, p. 374]. The moments of a 
BGD can be obtained by using the Taylor series expansion of the given Laplace transform. Hence, the mean $m_{i}$; variance $\sigma_{i}^{2}$, with $i=1,2$; covariance $\operatorname{cov}\left(x_{1}, x_{2}\right)$; and correlation coefficient $\operatorname{cor}\left(x_{1}, x_{2}\right)$ are expressed as

$$
\begin{aligned}
m_{i} & =\mathrm{E}\left[x_{i}\right]=q p_{i} \\
\sigma_{i}^{2} & =\mathrm{E}\left[\left(x_{i}-m_{i}\right)^{2}\right]=q p_{i}^{2} \\
\operatorname{cov}\left(x_{1}, x_{2}\right) & =\mathrm{E}\left[x_{1} x_{2}\right]-\mathrm{E}\left[x_{1}\right] \mathrm{E}\left[x_{2}\right] \\
& =q\left(p_{1} p_{2}-p_{12}\right) \\
\operatorname{cor}\left(x_{1}, x_{2}\right) & =\frac{\operatorname{cov}\left(x_{1}, x_{2}\right)}{\sigma_{1} \sigma_{2}}=\frac{p_{1} p_{2}-p_{12}}{p_{1} p_{2}} .
\end{aligned}
$$

\section{APPENDIX B \\ QUAD-POL TO HYBRID/COMPACT DUAL-POL DATA TRANSFORMATION}

In the bistatic scattering case, the scattering vector corresponding to the Sinclair matrix is defined as $\vec{k}=\left(S_{\mathrm{HH}}, S_{\mathrm{HV}}\right.$, $\left.S_{\mathrm{VH}}, S_{\mathrm{VV}}\right)^{T}$ [9]. In the monostatic case, under the scattering reciprocity and in the backscatter alignment convention, we have $S_{\mathrm{HV}}=S_{\mathrm{VH}}$ [34]. Therefore, the corresponding scattering vector is defined as $\underline{\vec{k}}=\left(S_{\mathrm{HH}}, \sqrt{2} S_{\mathrm{HV}}, S_{\mathrm{VV}}\right)^{T}$. The standard dual-pol scattering vectors $\vec{k}_{\mathrm{DP} 1}=\left(S_{\mathrm{HH}}, S_{\mathrm{HV}}\right)^{T}$, $\vec{k}_{\mathrm{DP} 2}=\left(S_{\mathrm{VH}}, S_{\mathrm{VV}}\right)^{T}$, and $\vec{k}_{\mathrm{DP} 3}=\left(S_{\mathrm{HH}}, S_{\mathrm{VV}}\right)^{T}$ are obtained straightforwardly from $\vec{k}$. Different hybrid/compact scattering vectors can be obtained from full polarimetric data. As an example, CL-pol mode [12] is further detailed. In CL-pol mode a right-circular illumination is achieved by $\boldsymbol{E}^{t}=1$ / $\sqrt{2}(1,-i)^{T} ; \quad$ thus, $\quad \boldsymbol{E}^{r}=1 / \sqrt{2}\left(S_{\mathrm{HH}}-i S_{\mathrm{HV}},-i S_{\mathrm{VV}}+\right.$ $\left.S_{\mathrm{HV}}\right)^{T}$. Hence, $\vec{k}_{\mathrm{CL}-\mathrm{pol}}=\left(E_{H}, E_{V}\right)^{T}$ where $E_{H}=(1,0) \boldsymbol{E}^{r}$ and $E_{V}=(0,1) \boldsymbol{E}^{r}$. Other compact scattering vectors can be derived in the same fashion. For more details, the readers are invited to see [9] and [12].

\section{ACKNOWLEDGMENT}

The authors acknowledge NASA JPL for providing AirSAR data, and MacDonald, Dettwiler and Associates for making available the RADARSAT-2 data used in this paper.

\section{REFERENCES}

[1] M. Cohen, "Radio astronomy polarization measurements," Proc. IRE, vol. 46, no. 1, pp. 172-183, Jan. 1958.

[2] J. Fritz and V. Chandrasekar, "Simultaneous observations and analysis of severe storms using polarimetric X-band SAR and ground-based weather radar," IEEE Trans. Geosci. Remote Sens., vol. 48, no. 10, pp. 3622-3637, Oct. 2010

[3] M. Galletti, D. H. O. Bebbington, M. Chandra, and T. Borner, "Measurement and characterization of entropy and degree of polarization of weather radar targets," IEEE Trans. Geosci. Remote Sens., vol. 46, no. 10, pp. 3196-3207, Oct. 2008

[4] R. Touzi, A. Deschamps, and G. Rother, "Phase of target scattering for wetland characterization using polarimetric C-band SAR," IEEE Trans. Geosci. Remote Sens., vol. 47, no. 9, pp. 3241-3261, Sep. 2009.

[5] T. L. Toan, A. Beaudoin, J. Riom, and D. Guyon, "Relating forest biomass to SAR data," IEEE Trans. Geosci. Remote Sens., vol. 30, no. 2, pp. 403411, Mar. 1992.
[6] M. Migliaccio, A. Gambardella, F. Nunziata, M. Shimada, and O. Isoguchi, "The PALSAR polarimetric mode for sea oil slick observation," IEEE Trans. Geosci. Remote Sens., vol. 47, no. 12, pp. 4032-4041, Dec. 2009.

[7] L. B. Wolff, "Polarization camera for computer vision with a beam splitter," J. Opt. Soc. Amer. A, vol. 11, no. 11, Nov. 1994

[8] S. L. Jacques, J. C. Ramella-Roman, and K. Lee, "Imaging skin pathology with polarized light," J. Biomed. Opt., vol. 7, no. 3, pp. 329-340, 2002.

[9] J.-S. Lee and E. Pottier, Polarimetric Radar Imaging: From Basics to Applications. Boca Raton, FL: CRC Press, 2009.

[10] J.-C. Souyris, P. Imbo, R. Fjortoft, S. Mingot, and J.-S. Lee, "Compact polarimetry based on symmetry properties of geophysical media: The $\pi / 4$ mode," IEEE Trans. Geosci. Remote Sens., vol. 43, no. 3, pp. 634-646, Mar. 2005.

[11] N. Stacy and M. Preiss, "Compact polarimetric analysis of X-band SAR data," in Proc. EUSAR, Germany, May 2006.

[12] R. Raney, "Hybrid-polarity SAR architecture," IEEE Trans. Geosci. Remote Sens., vol. 45, no. 11, pp. 3397-3404, Nov. 2007.

[13] V. B. Taylor, "Cyclops: The JPL Airsar synoptic processor," in Proc. IGARSS'92, Houston, TX, May 1992, pp. 652-654.

[14] M. Nord, T. Ainsworth, J.-S. Lee, and N. Stacy, "Comparison of compact polarimetric synthetic aperture radar modes," IEEE Trans. Geosci. Remote Sens., vol. 47, no. 1, pp. 174-188, Jan. 2009.

[15] M.-L. Truong-Loi, A. Freeman, P. Dubois-Fernandez, and E. Pottier, "Estimation of soil moisture and faraday rotation from bare surfaces using compact polarimetry," IEEE Trans. Geosci. Remote Sens., vol. 47, no. 11 , pp. 3608-3615, Nov. 2009.

[16] R. C. Jones, "A new calculus for the treatment of optical systems: A more general formulation and description of another calculus," J. Opt. Soc. Amer, vol. 37, no. 2, pp. 107-110, 1947.

[17] E. Wolf, "Coherence properties of partially polarized electromagnetic radiation," Nuovo Cim., vol. 13, no. 6, pp. 1165-1181, Sep. 1959.

[18] J. W. Goodman, Statistical Optics. New York: Wiley, 1985.

[19] J. S. Lee, K. W. Hoppel, S. A. Mango, and A. R. Miller, "Intensity and phase statistics of multilook polarimetric and interferometric SAR imagery," IEEE Trans. Geosci. Remote Sens., vol. 32, no. 5, pp. 10171028, Sep. 1994.

[20] R. Touzi and A. Lopes, "Statistics of the stokes parameters and of the complex coherence parameters in one-look and multilook speckle fields," IEEE Trans. Geosci. Remote Sens., vol. 34, no. 2, pp. 519-531, Mar. 1996.

[21] R. C. Jones, "A new calculus for the treatment of optical systems," J. Opt Soc. Amer, vol. 31, no. 7, pp. 488-493, Jul. 1941.

[22] N. Wiener, "Generalized harmonic analysis," Acta Math., vol. 55, pp. 117-258, 1930.

[23] G. G. Stokes, "On the composition and resolution of streams of polarized light from different sources," Trans. Camb. Phil. Soc., vol. 9, pp. 399-416, 1852.

[24] P. E. Green, Jr., "Radar measurements of target scattering properties," in Radar astronomy, J. V. Evans and T. Hagfors, Eds. New York: McGrawHill, 1968, pp. 1-78.

[25] J. R. Huynen, "Phenomenological theory of radar targets," Ph.D. dissertation, Delft Univ. Technol., Delft, The Netherlands, Dec., 1970.

[26] M. Born, E. Wolf, and A. Bhatia, Principles of Optics: Electromagnetic Theory of Propagation, Interference and Diffraction of Light. Cambridge, U.K.: Cambridge Univ. Press, 1999.

[27] A. Guissard, "Mueller and Kennaugh matrices in radar polarimetry," IEEE Trans. Geosci. Remote Sens., vol. 32, no. 3, pp. 590-597, May 1994.

[28] C. Brosseau, Fundamentals of Polarized Light: A Statistical Optics Approach. Hoboken, NJ: Wiley, 1998.

[29] S. N. Anfinsen, A. P. Doulgeris, and T. Eltoft, "Estimation of the equivalent number of looks in polarimetric synthetic aperture radar imagery," IEEE Trans. Geosci. Remote Sens., vol. 47, no. 11, pp. 3795-3809, Nov. 2009.

[30] F. Chatelain, J.-Y. Tourneret, M. Roche, and M. Alouini, "Estimating the polarization degree of polarimetric images in coherent illumination using maximum likelihood methods," J. Opt. Soc. Amer. A, vol. 26, no. 6, pp. 1348-1359, 2009

[31] R. Shirvany, M. Chabert, and J.-Y. Tourneret, "Ship and oil-spill detection using the degree of polarization in linear and hybrid/compact dual-pol SAR," 2012, to be published.

[32] M. Abramowitz and I. A. Stegun, Handbook of Mathematical Functions. New York: Dover, 1965.

[33] M. Roche, J. Fade, and P. Réfrégier, "Parametric estimation of the square degree of polarization from two intensity images degraded by fully developed speckle noise," J. Opt. Soc. Amer. A, vol. 24, no. 9, pp. 2719-2727, 2007 . 
[34] S. V. Nghiem, S. H. Yueh, R. Kwok, and F. K. Li, "Symmetry properties in polarimetric remote sensing," Radio Sci., vol. 27, pp. 693-711, Oct. 1992.

[35] J. S. Lee, M. R. Grunes, and E. Pottier, "Quantitative comparison of classification capability: fully polarimetric versus dual and single-polarization SAR," IEEE Trans. Geosci. Remote Sens., vol. 39, no. 11, pp. 2343-2351, Nov. 2001.

[36] N. R. Goodman, "Statistical analysis based on a certain multivariate complex Gaussian distribution (an introduction)," Ann. Math. Stat., vol. 34, no. 1, pp. 152-177, 1963.

[37] P. Bernardoff, "Which multivariate gamma distributions are infinitely divisible?" Bernoulli, vol. 12, no. 1, 2006.

[38] G. Letac and J. Wesolowski, "Laplace transforms which are negative powers of quadratic polynomials," Trans. AMS, vol. 360, no. 12, pp. 64756496, Dec. 2008.

[39] F. Chatelain, J.-Y. Tourneret, J. Inglada, and A. Ferrari, "Bivariate gamma distributions for image registration and change detection," IEEE Trans. Image Process., vol. 16, no. 7, pp. 1796-1806, Jul. 2007.

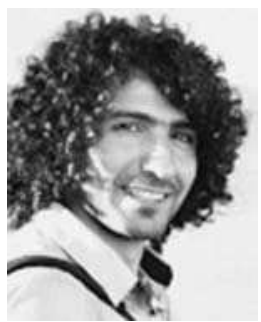

Reza Shirvany (S'06) received the M.E. degree (diplôme d'ingénieur) in electrical engineering from the École Nationale Supérieure d'Électronique, d'Électrotechnique, d'Informatique, d'Hydraulique, et des Télécommunications (ENSEEIHT), Toulouse, France, and the M.Sc. degree in signal and image processing from Institut National Polytechnique de Toulouse (INPT), Toulouse, both in 2008. He is currently working toward the Ph.D. degree at the University of Toulouse, Toulouse.

He was a Research Intern with the Biomedical Imaging Group, École Polytechnique Fédérale de Lausanne (EPFL), Lausanne, Switzerland, working on B-splines and wavelets. His main research interest include mathematical modeling and applications of polarimetric imagery.

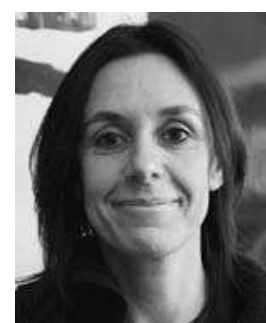

Marie Chabert (M'10) received the Eng. degree in electronics and signal processing from the ENSEEIHT, Toulouse, France, in 1994 and the M.Sc. degree in signal processing, the Ph.D. degree in signal processing, and the Habilitation a Diriger les Recherches (HDR) from the National Polytechnic Institute of Toulouse, Toulouse, in 1994, 1997, and 2007 , respectively.

She is currently an Associate Professor of signal and image processing. She is with the engineering school INPT-ENSEEIHT, part of the University of Toulouse. She is conducting her research with the Signal and Communication Team of the Institut de Recherche en Informatique de Toulouse (UMR 5505 of the CNRS). Her research interests include nonuniform sampling, timefrequency diagnosis and condition monitoring, and statistical modeling of heterogeneous data in remote sensing.

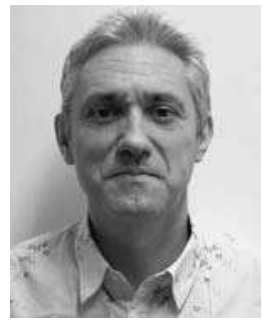

Jean-Yves Tourneret (SM'08) received the Ingénieur degree in electrical engineering from the École Nationale Supérieure d'Électronique, d'Électrotechnique, d'Informatique, d'Hydraulique, et des Télécommunications (ENSEEIHT), Toulouse, France, in 1989 and the Ph.D. degree from the National Polytechnic Institute, Toulouse, in 1992.

$\mathrm{He}$ is currently a Professor with the University of Toulouse (ENSEEIHT) and a member of the IRIT Laboratory (UMR 5505 of the CNRS). His research interests include statistical signal processing, with a particular interest to Bayesian methods and Markov chain Monte Carlo algorithms.

Dr. Tourneret was the Program Chair of the European Conference on Signal Processing held in Toulouse in 2002. He was also member of the Organizing Committee for the International Conference ICASSP'06 held in Toulouse in 2006. He has been a member of different technical committees including the Signal Processing Theory and Methods Committee of the IEEE Signal Processing Society from 2001 to 2007 and from 2010 up to present. $\mathrm{He}$ served as an Associate Editor for the IEEE TRANSACTIONS ON SignaL PROCESSING from 2008 to 2011. 